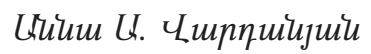

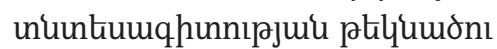

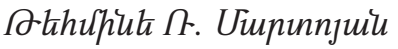

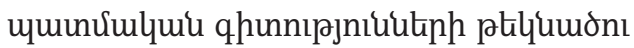

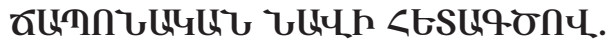

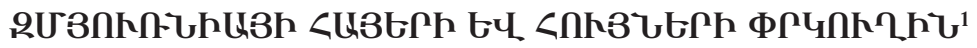

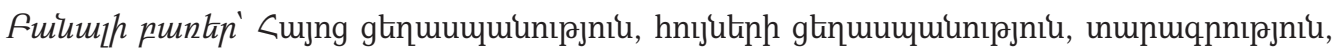

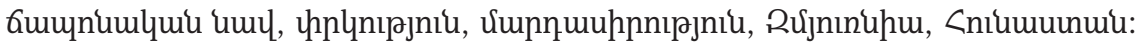

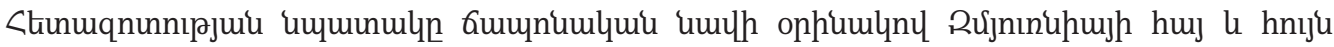

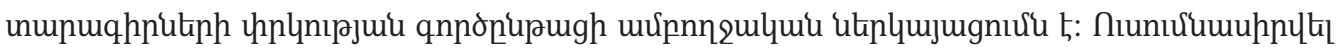

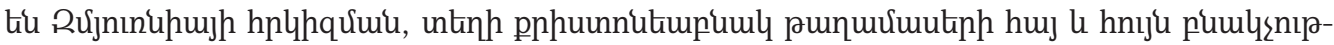

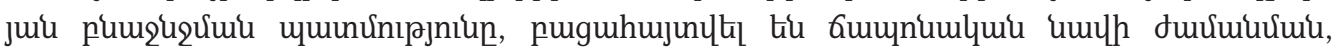

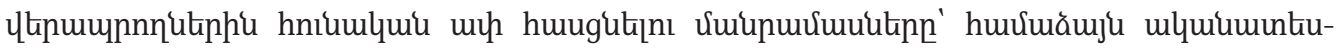

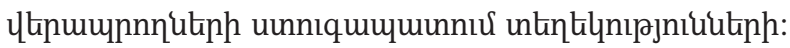

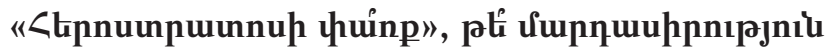

hंus

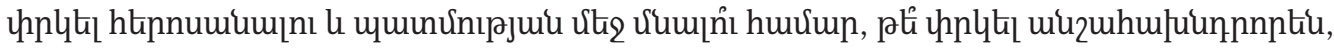

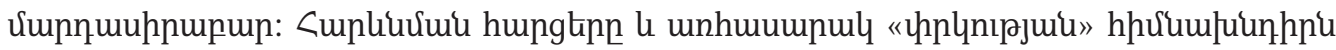

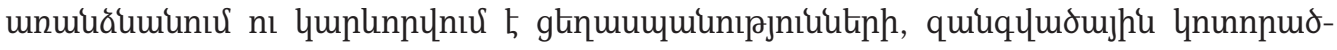

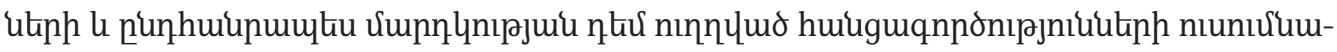
uppnıрjuiu huर्umuntpuinnıर:

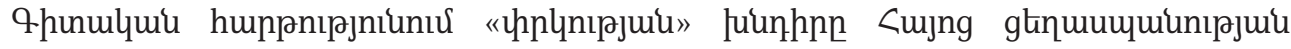

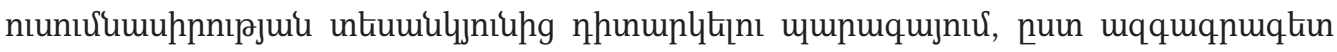

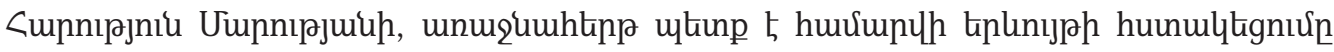

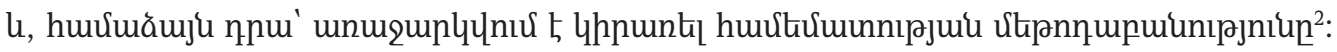

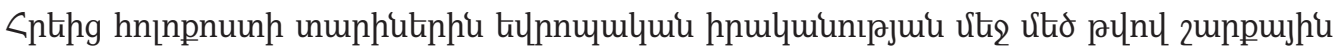

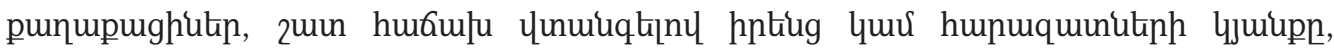

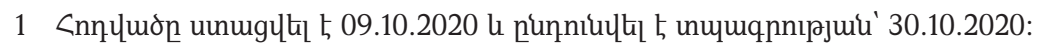

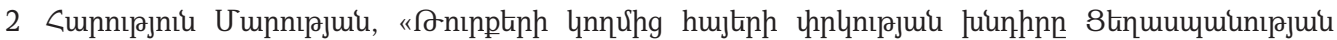

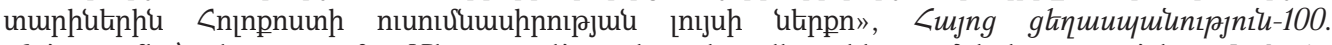

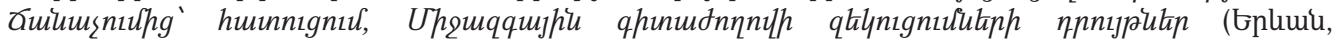

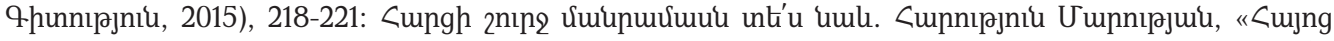

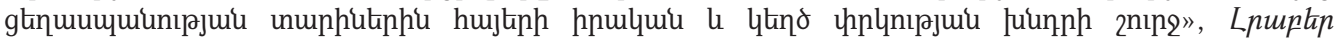

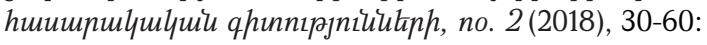




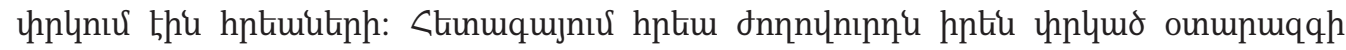

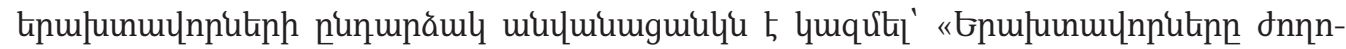
unıpnutph/Sumplquing Utig ("The Righteous Among the Nations")

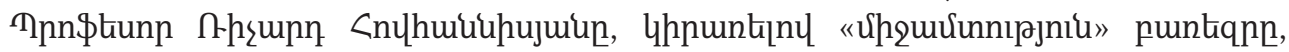

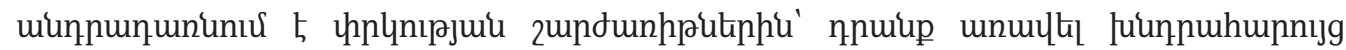

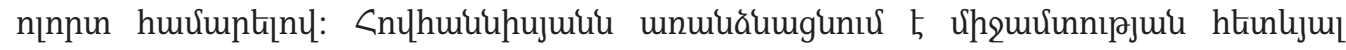

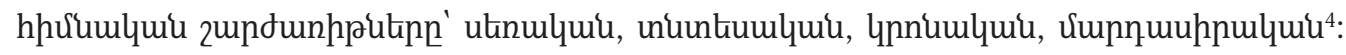

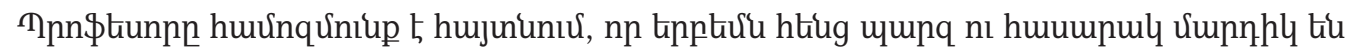

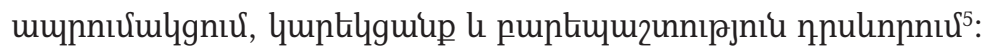

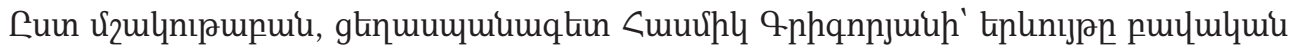

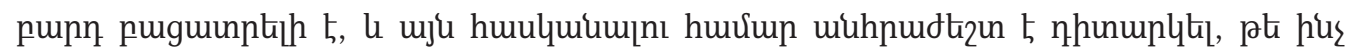

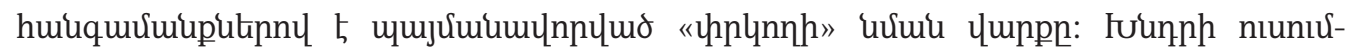

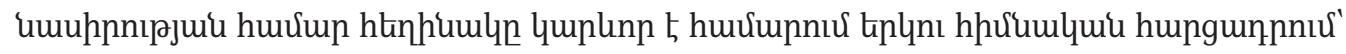

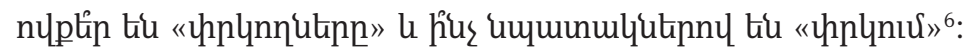

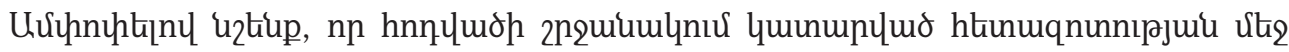

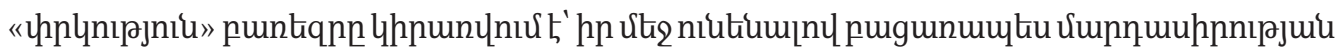

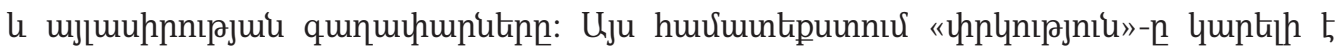

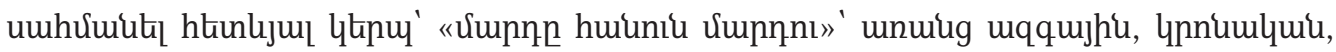

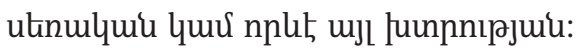

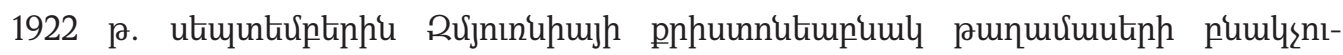

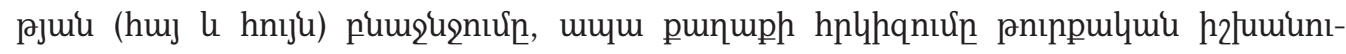

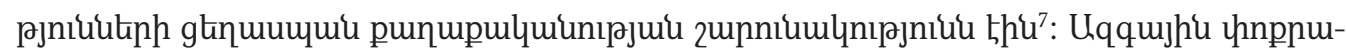

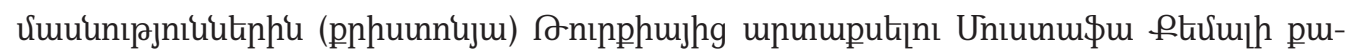

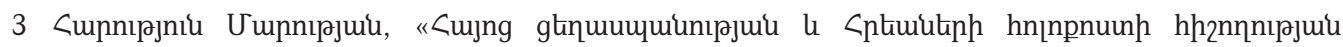

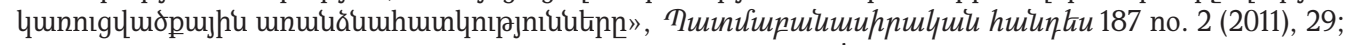
http://www. yadvashem.org//yv/en/righteous/about.asp, nhunlthl h' 25.08.2020:

4 Richard Hovannisian, "Intervention and Shades of Altruism during the Armenian Genocide," The Armenian Genocide, History, Politics, Ethics, ed. Richard Hovannisian (New York: St. Martin's Press, 1992), 179.

5 unıju untinnux:

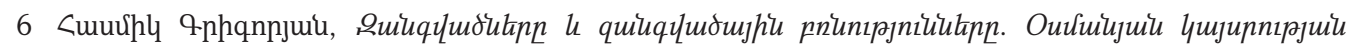

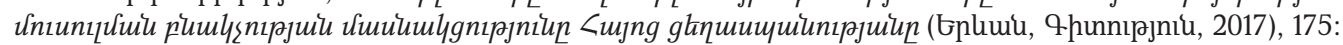

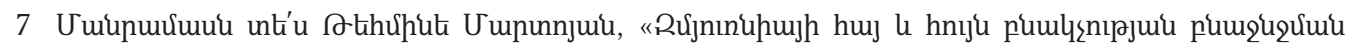

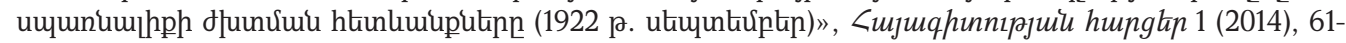
75; Tehmine Martoyan, "The Destruction of Smyrna in 1922: An Armenian and Greek Shared Tragedy," Genocide in the Ottoman Empire, Armenians, Assyrians, and Greeks, 1913-1923, ed. George N. Shirinian (New York: Asia Minor and Pontos Hellenic Research Center, 2017), 227-249; Tehmine Martoyan, "Denying the Possibility of Annihilation during Genocide: A Case Study of the Armenians and Greeks, 1915-1922," New Perspectives on the Genocide of the Greeks in the Ottoman Empire, 1913-1923, ed. George N. Shirinian (Chicago, IL: Asia Minor and Pontos Hellenic Research Center, 2019), 253-268. 


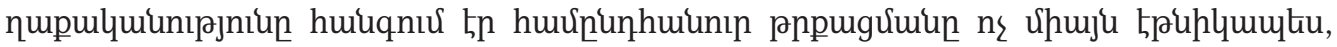
ujl «щumuर्umuminntiu»:

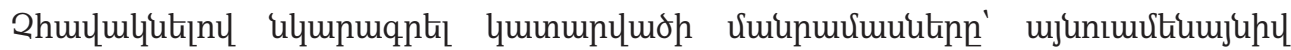

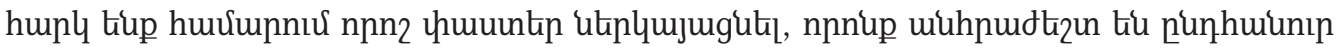

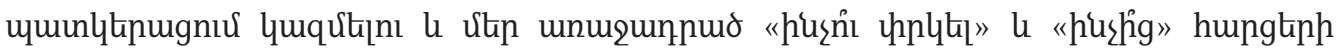

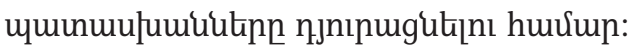

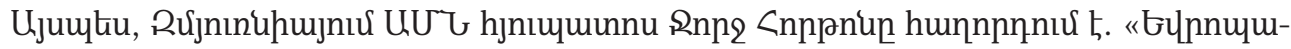

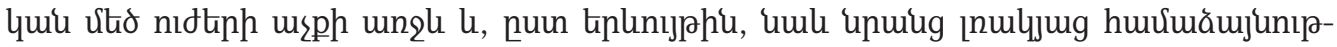

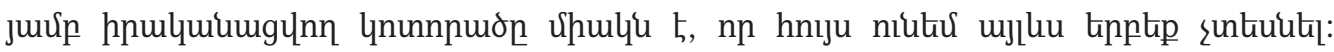

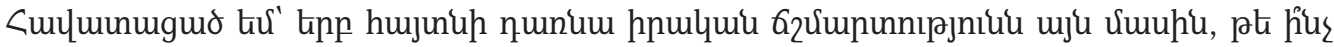

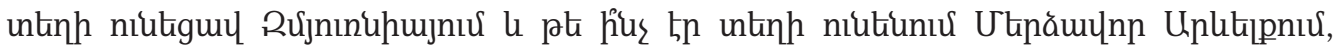

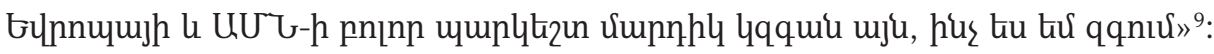

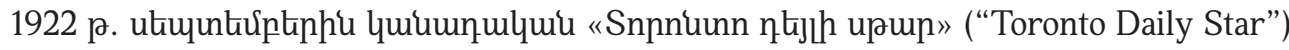

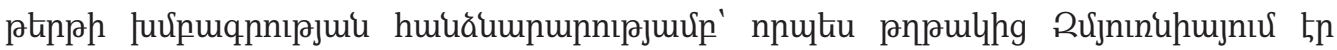

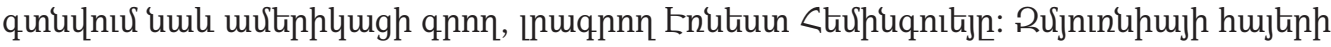

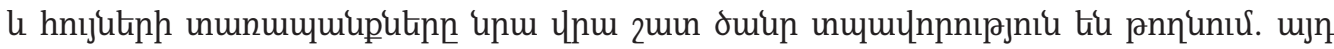

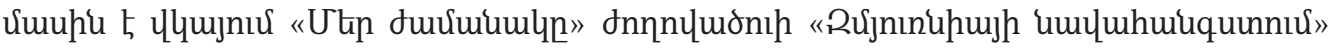

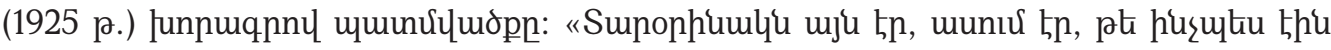

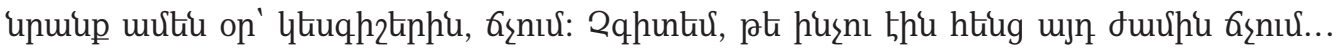

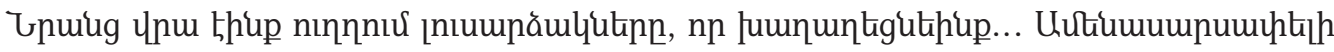

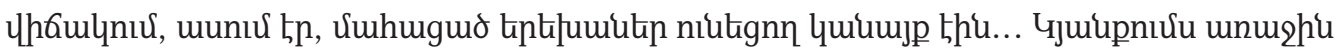

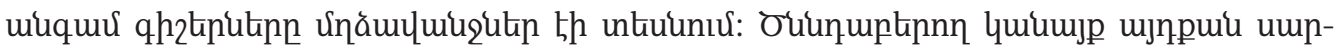

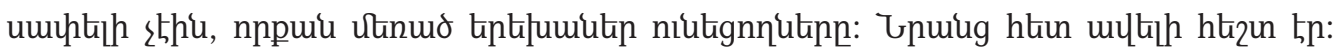

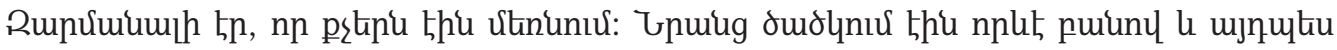

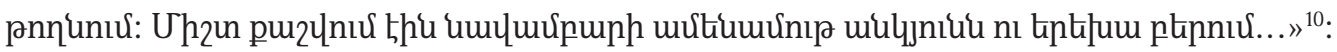

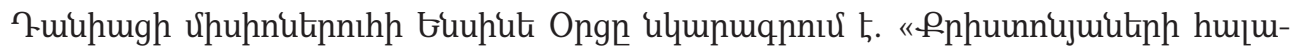

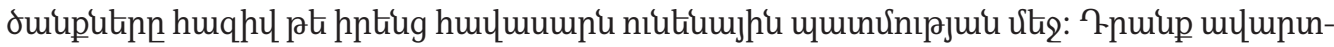

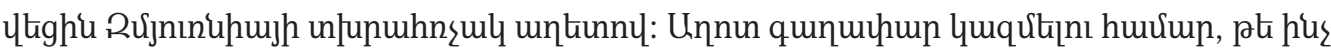

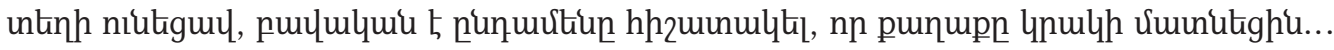

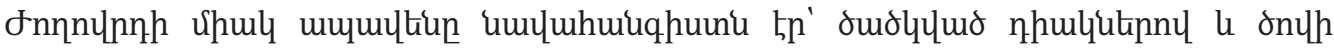
unjniumutinl 2nnu»"

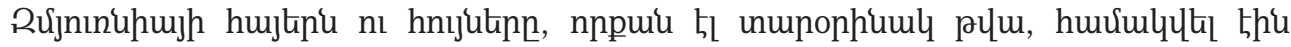

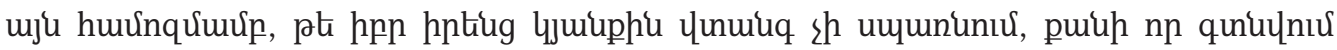

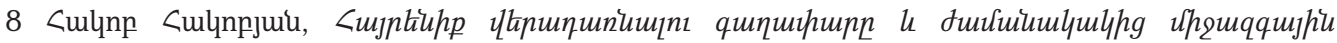
hruiniupn (Gpluwu, Uunnhl, 2000), 114:

9 George Horton, Report on Turkey: USA Consular Documents (Athens: Journalists' Union of the Athens Daily Newspapers, 1985), 24.

10 Ernest Hemingway, In Our Time (New York: Scribner, 1930), 11-12.

11 Jensine Oerts Peters, Tests and Triumphs of Armenians in Turkey and Macedonia (USA: Zondervan, 1940), 48. 


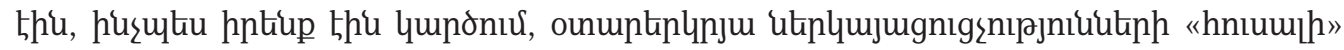
hnumunt ukppn:

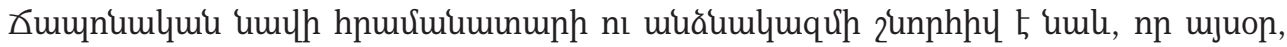

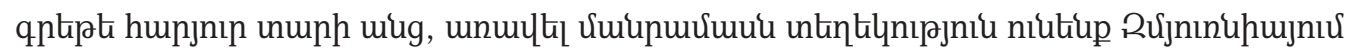

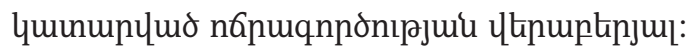

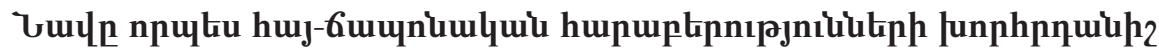

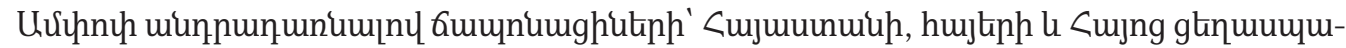

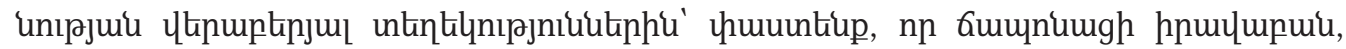

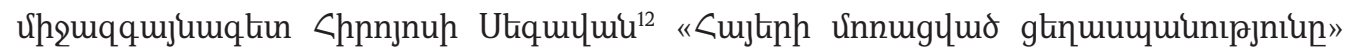

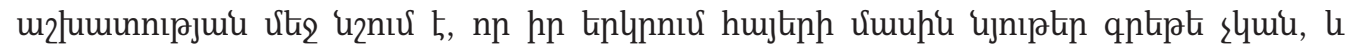

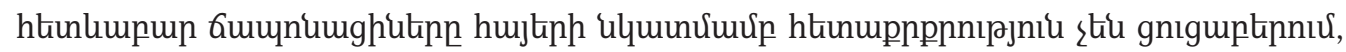

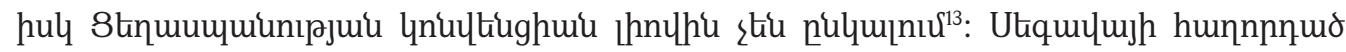

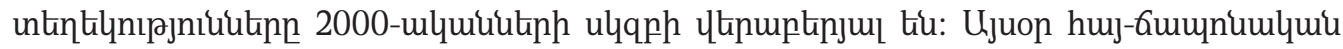

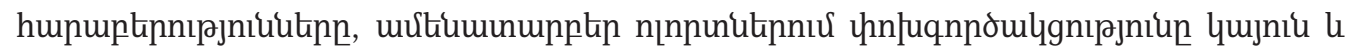
hinuulyunujhu tiu:

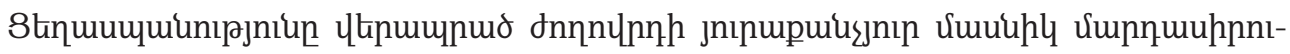

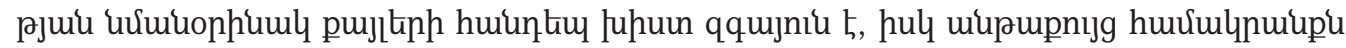

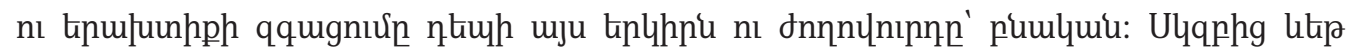

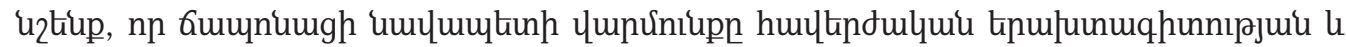

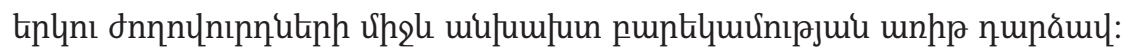

\section{Фplnıpjuiu Junphnıpn}

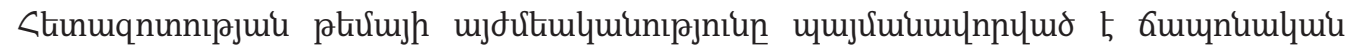

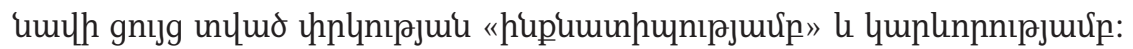

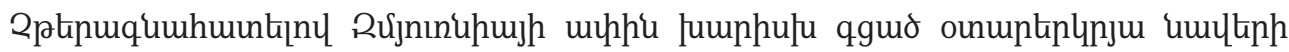

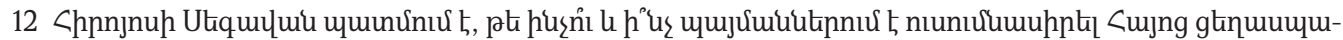

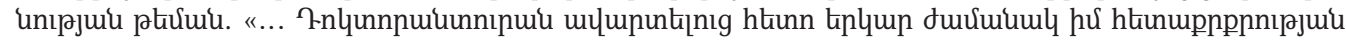

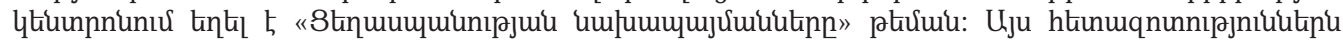

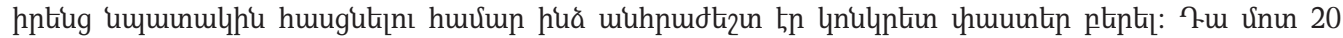

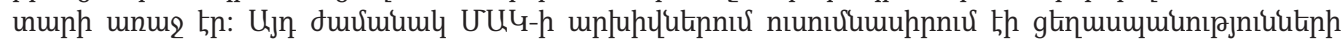

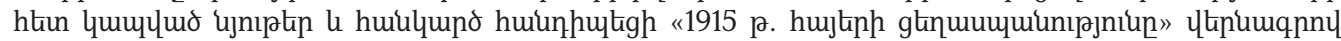

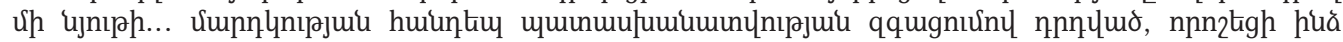

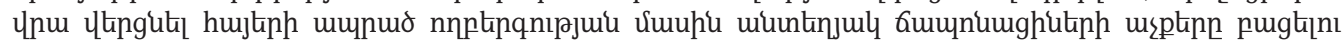

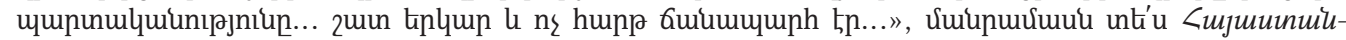

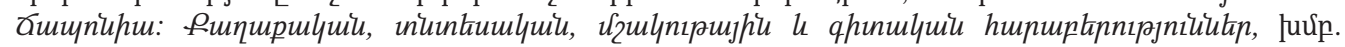

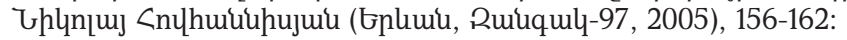

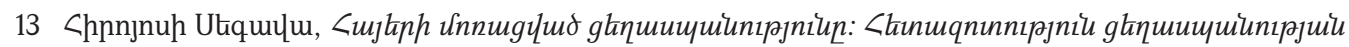

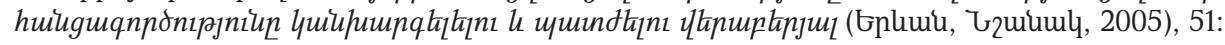




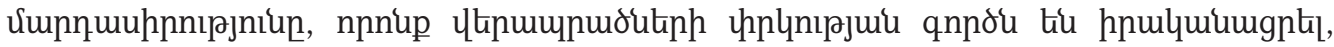

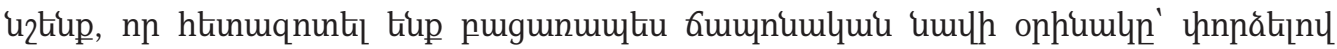

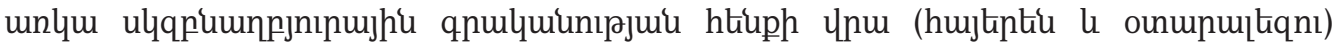

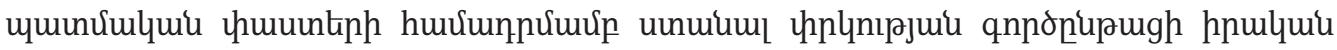

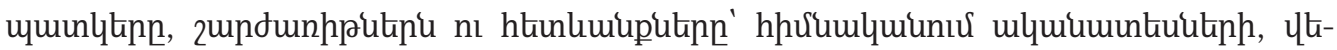

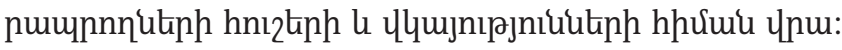

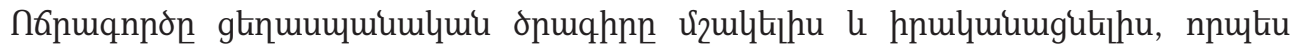

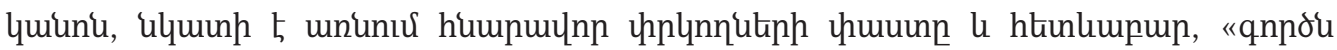

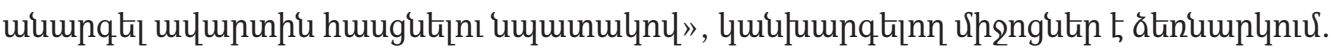

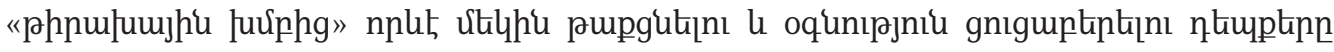

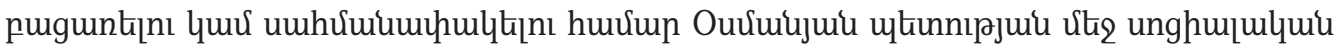

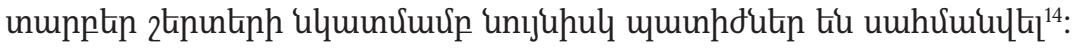

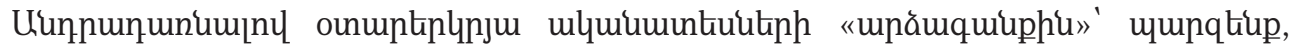

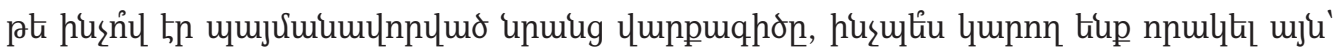

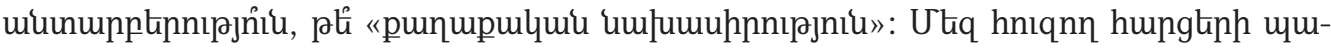

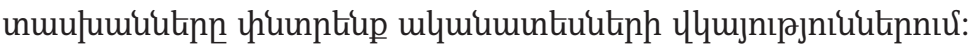

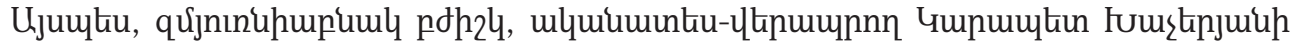

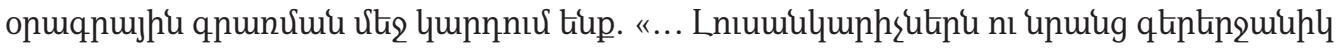

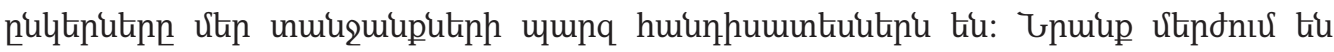

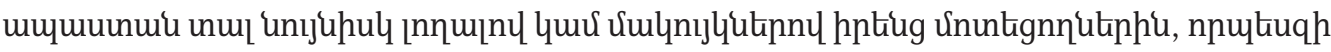

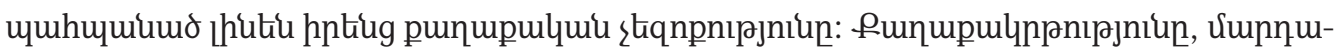

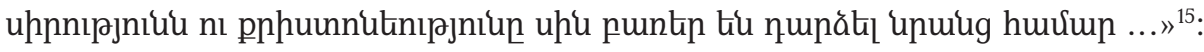

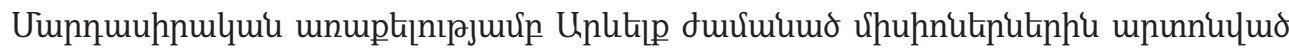

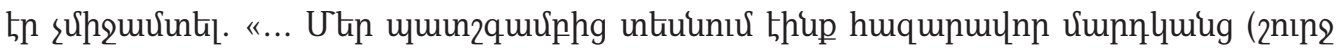

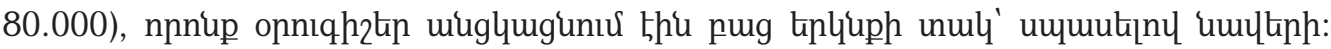

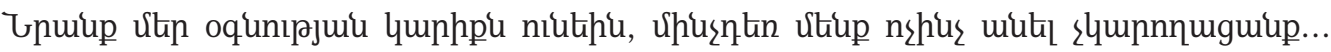

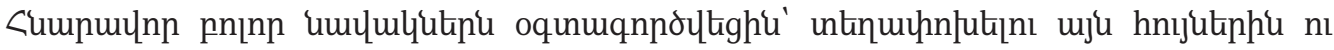

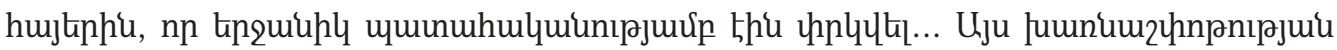
Utiq mưnnmuium

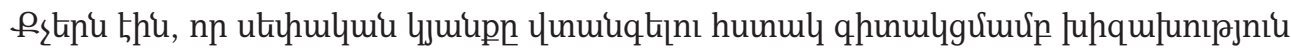

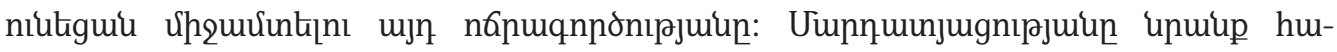

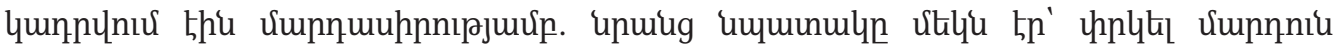

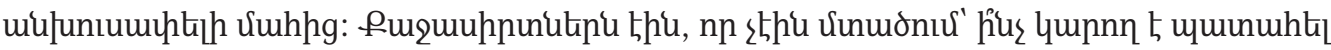

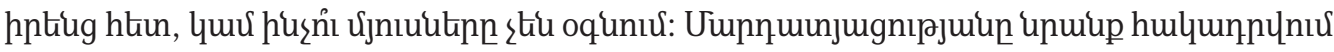
thu umpnumunnıрjứp:

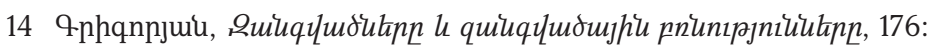

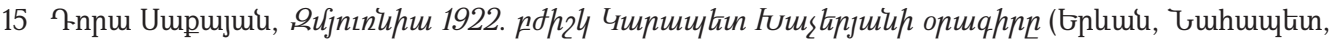
2005), 78:

16 Peters, Tests and Triumphs, 48-49: 


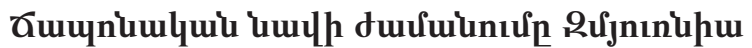

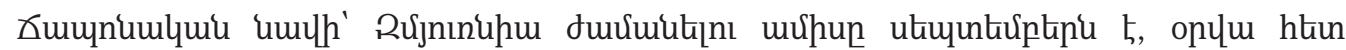

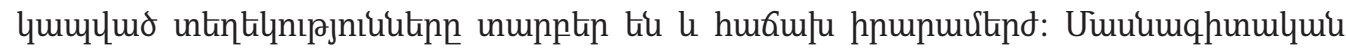

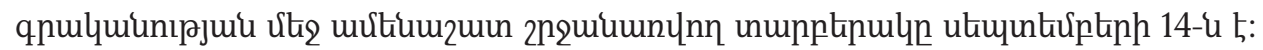

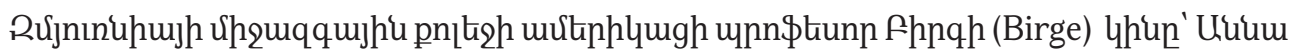

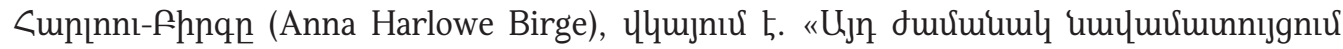

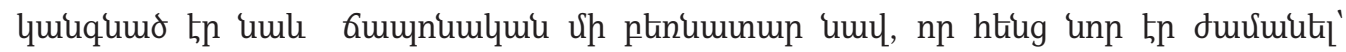

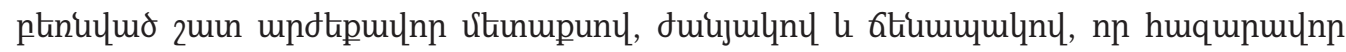

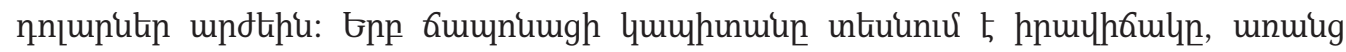

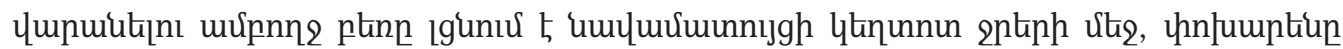

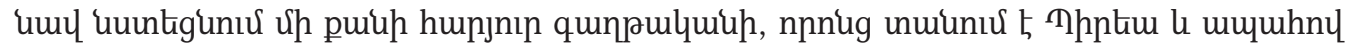

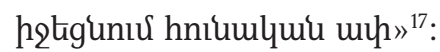

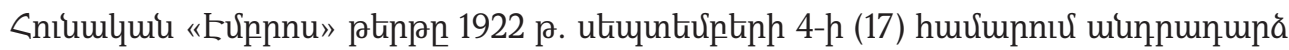

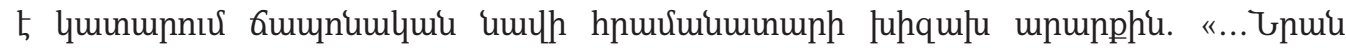

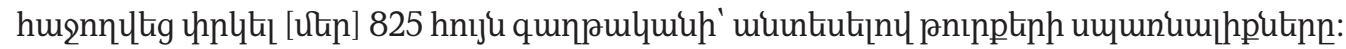

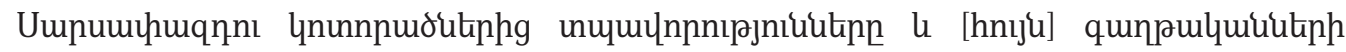

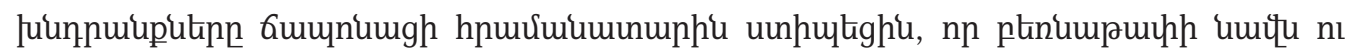

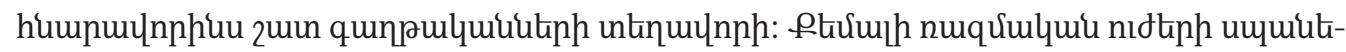

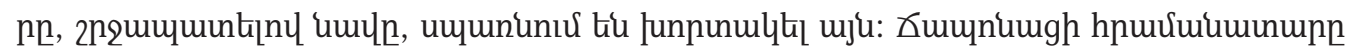

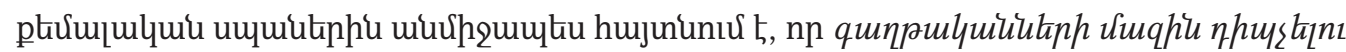

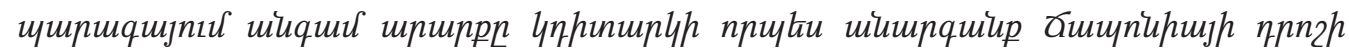

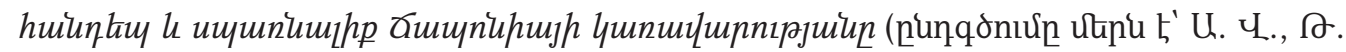

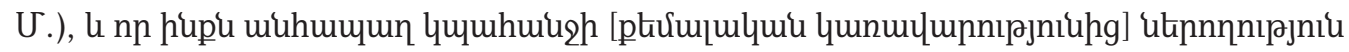

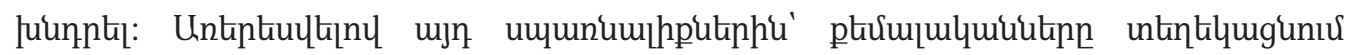

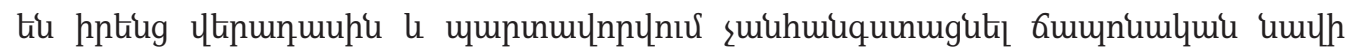
quinpulquiutinhu» ${ }^{18}$ :

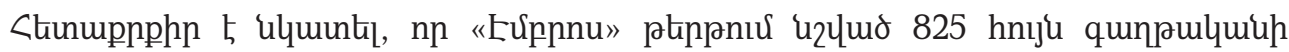

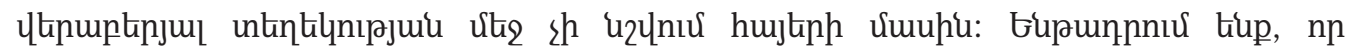

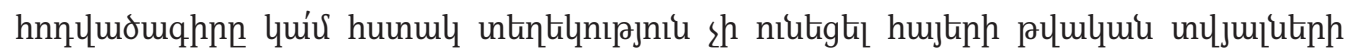

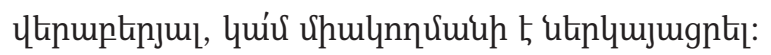

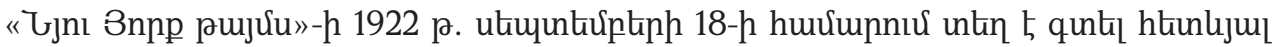

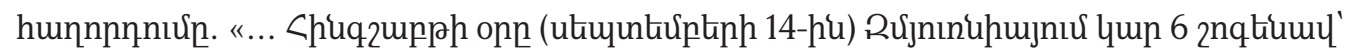

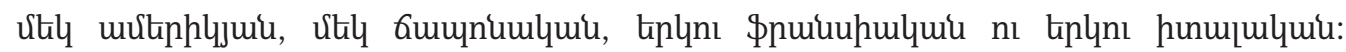

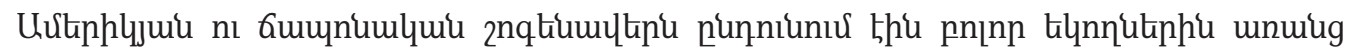

17 "Japanese at Smyrna," Boston Globe (Boston), 3 December 1922, hnnưu nuun' Stavros Stavridis, "Special to the National Herald," at http://www.greece.org/main/index.php?option=com_content\&id=59\&Itemid=82, nhunцlı 5 06.10.2020:

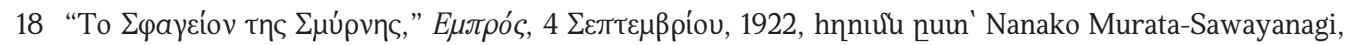
"The Memory in a Crisis: A Japanese Ship Helping Out Greek Refugees on the Quay of Smyrna in 1922," at https://hermes-ir.lib.hit-u.ac.jp/hermes/ir/re/28545/?lang=1, nhunltkl 5 06.10.2020: 


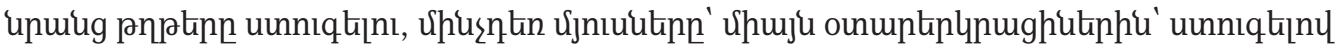

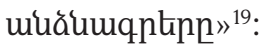

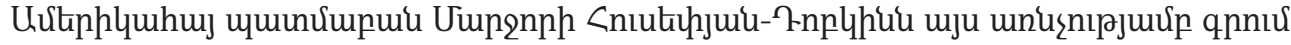

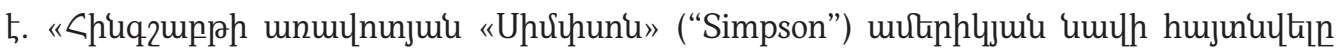

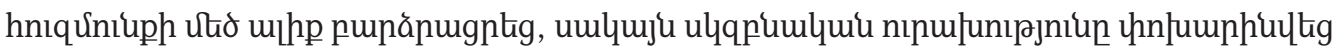

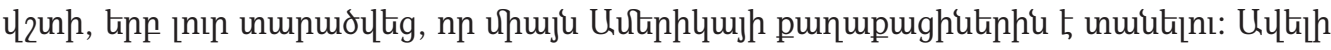

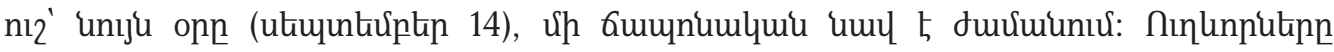

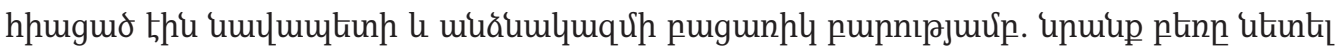

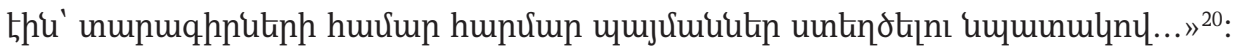

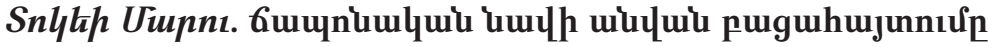

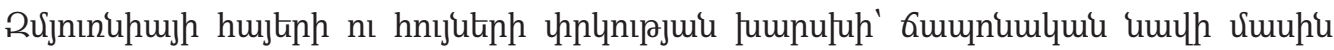

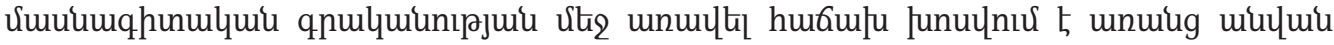
hhruunulynipjuiu:

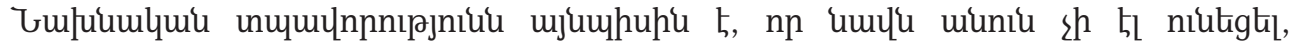

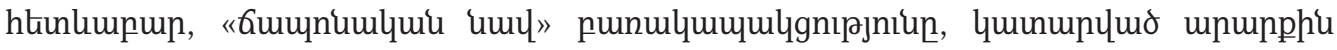

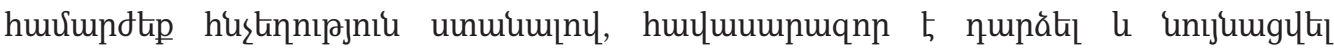

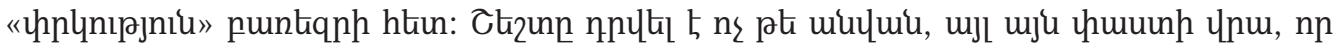

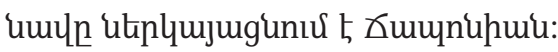

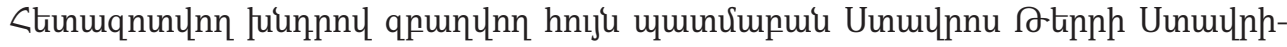

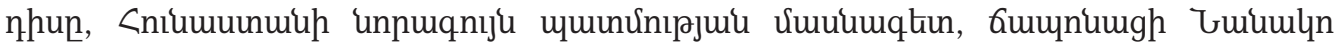

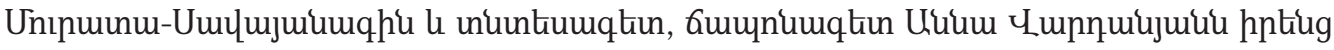

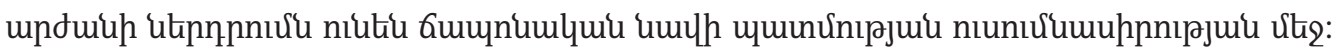

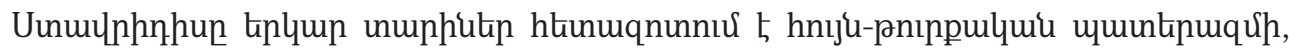

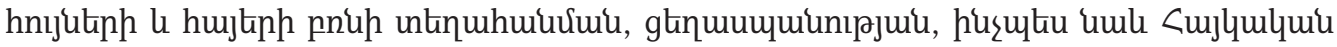

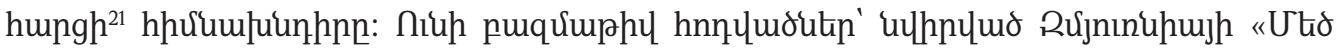

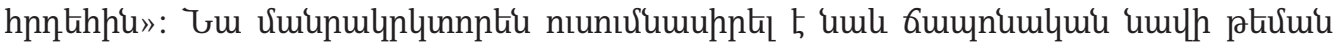

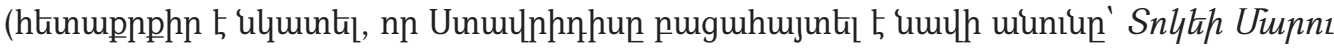

19 “Smyrna's Ravagers Fired on Americans," New York Times (New York), 18 September 1922, at https:// www.nytimes.com/1922/09/18/archives/smyrnas-ravagers-fired-on-americans-ymca-workers-were-heldup-and.html, nhunцtil 5 06.10.2020:

20 Marjorie Housepian Dobkin, Smyrna 1922: the Destruction of a City (New York, Newmark, 1998), 174. Uhưhunup Suuhu unt'u umu American Accounts Documenting the Destruction of Smyrna by the Kemalist Turkish Forces, September 1922, ed. by Constantine G. Hatzidimitriou (New York: Melissa International, 2005), 67.

21 Terry Stavridis, The Armenian Question 1918-1920, https://www.researchgate.net publication/279556219_ The_Armenian_Question_1918-20, nhunцtㅣㄴ 5 06.10.2020: 


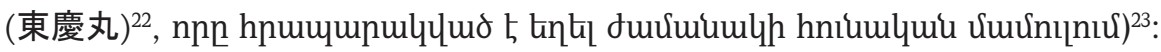

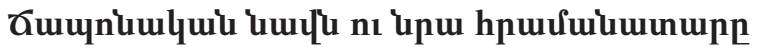

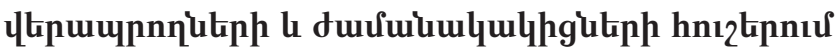

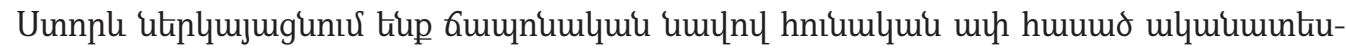

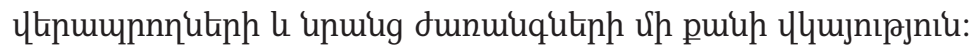

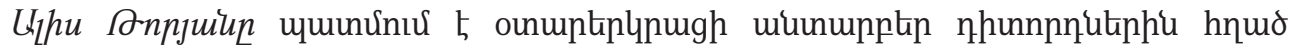

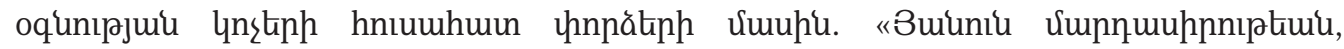

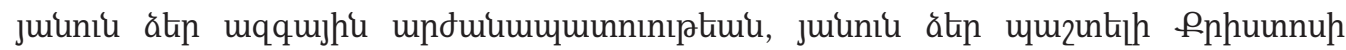

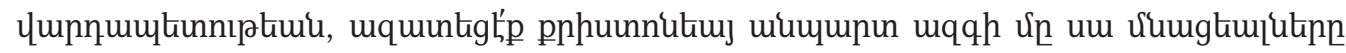

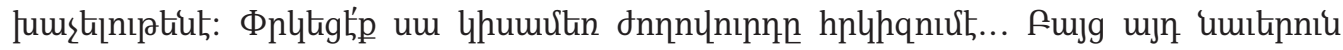

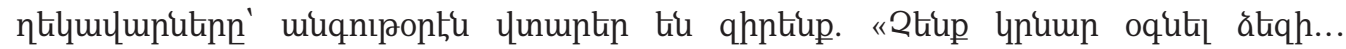

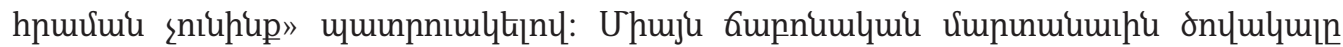

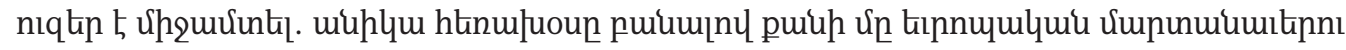

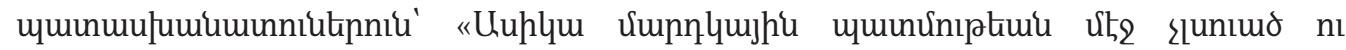

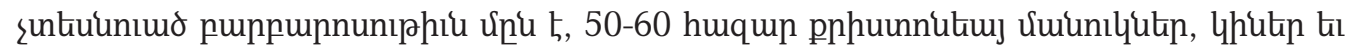

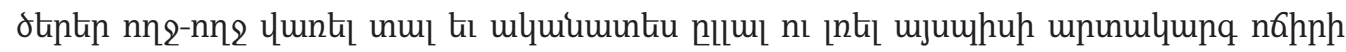

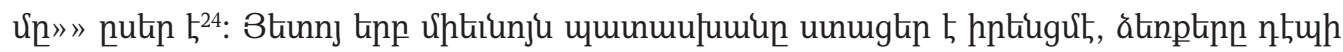

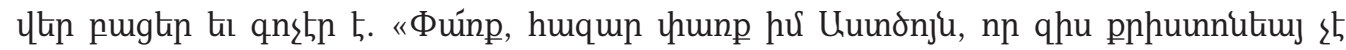
১uư ătiqh uŁu» ${ }^{25}$ :

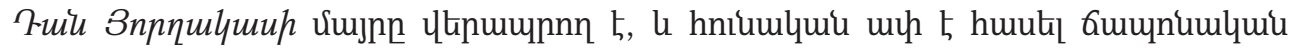

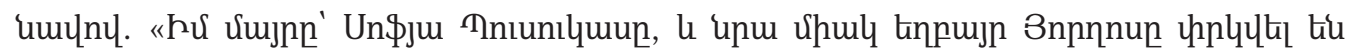

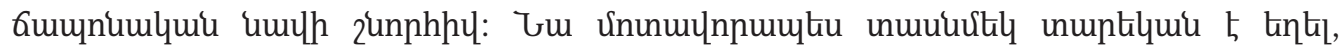

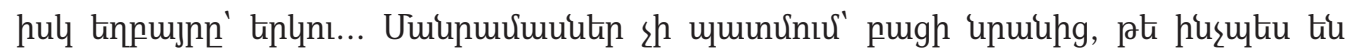

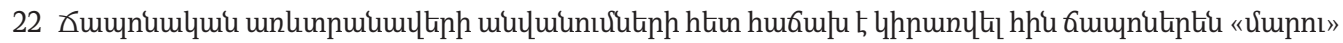

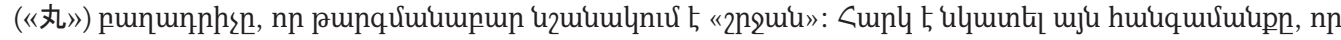

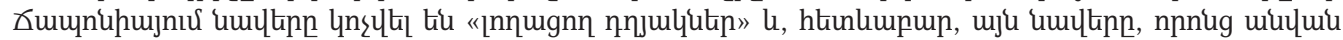

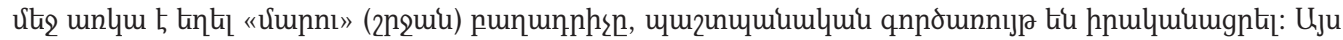

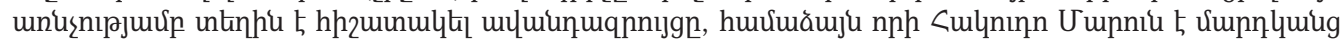

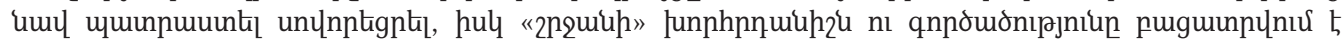

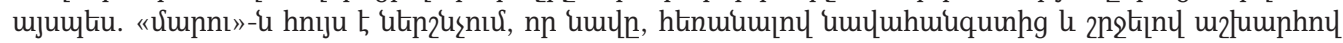

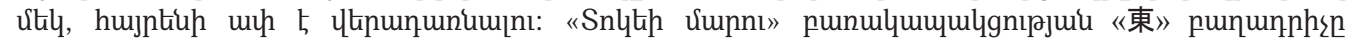

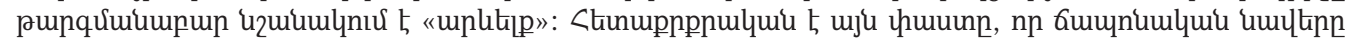

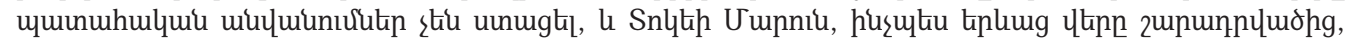

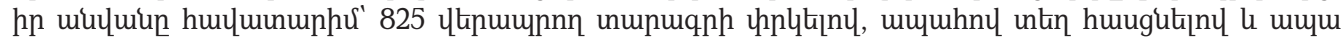

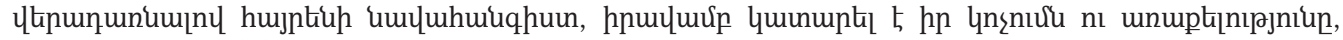

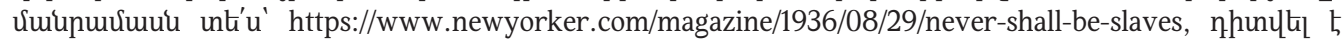
12.10.2020:

23 Terry Stawridis, “Japanese at Smyrna: September 1922,” AHIF Policy Jounrnal 7 (2016), 1-5.

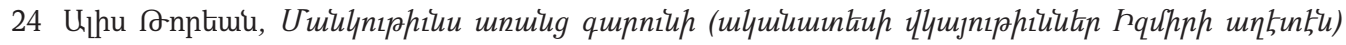
(Thјрnıр, Uипщuи, 1975), 75:

25 unıju unknnıx: 


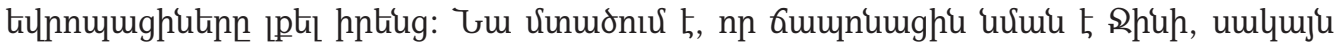

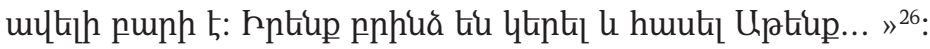

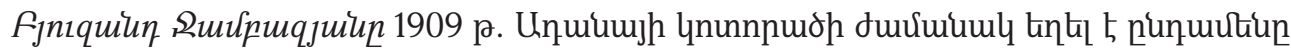

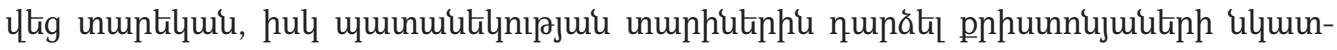

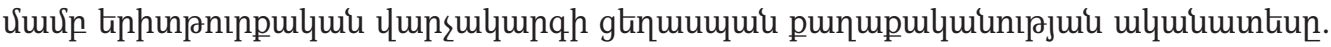

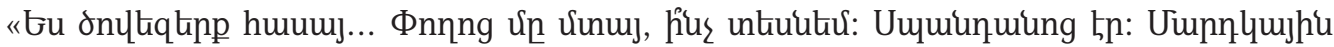

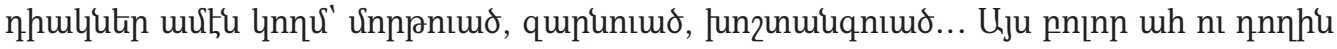

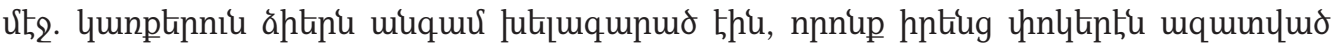

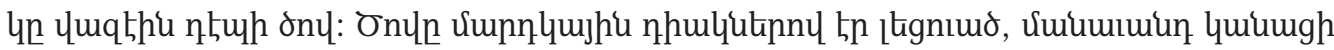

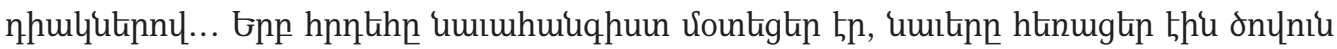

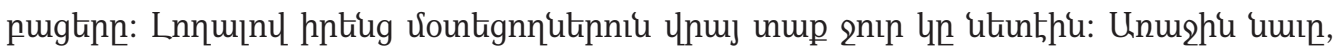

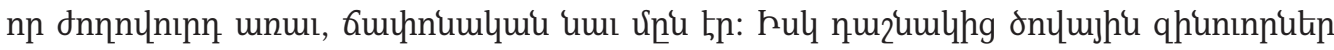

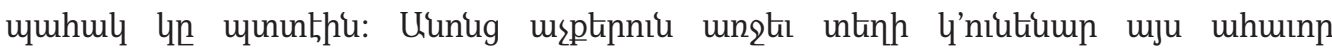

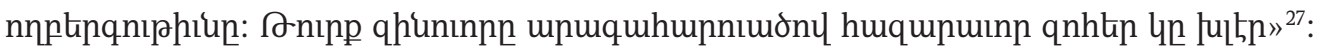

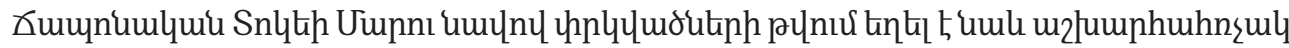

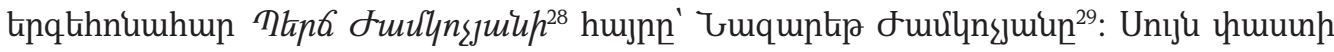

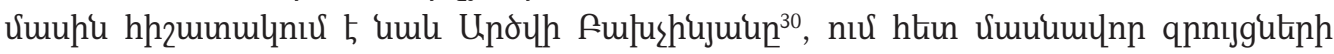

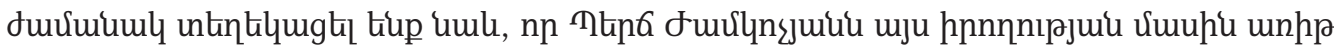

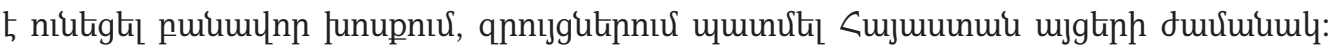

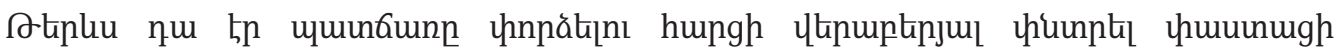

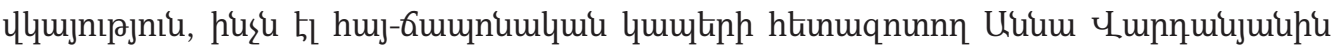

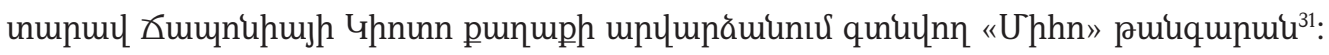

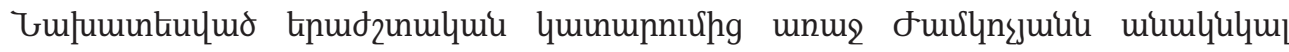

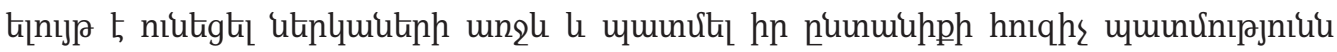

26 Constantine G. Hatzidimitriou, "The Genocide of Anatolian Christians and the Destruction of Smyrna: Scholarship and the Evidence, Ninety Years Later," at https://hellenicresearchcenter.org/education/ lectures-2/destruction-of-smyrna/, nhunltil 5 06.10.2020:

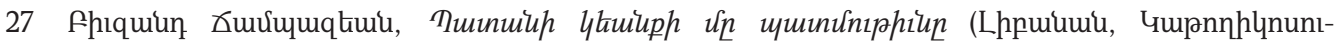
ptiuk <ujng Utidh Suiu Ghıhlhnj, 1960), 48:

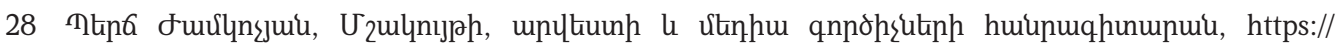
avproduction. am/? $\mathrm{ln}=$ am\&page=person\&id=4091\&name=\%D5\%8A\%D5\%A5\%D6\%80\%D5\%B3_\%D4\%BA\%D5 \%A1\%D5\%B4\%D5\%AF\%D5\%B8\%D5\%B9\%D5\%B5\%D5\%A1\%D5\%B6, nhunцti t5 09.09.2020:

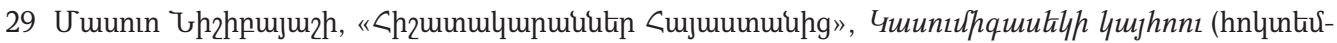
ptip, 2004), 14 (6umunutiptiu):

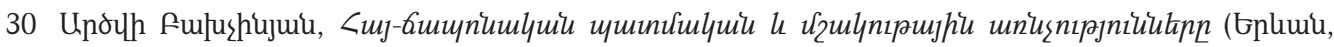
hinhiumlumjhu hpuinumulnnıjniu, 2018), 61:

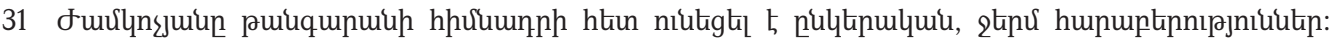

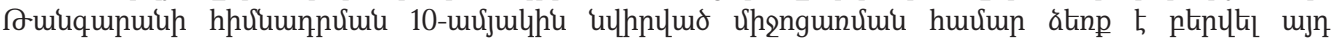

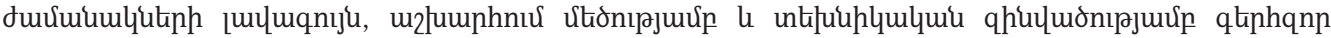

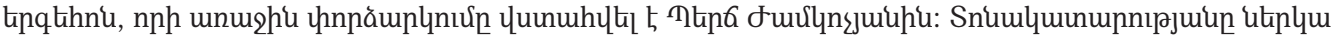

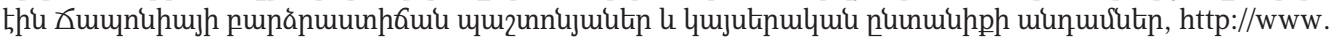
miho.or.jp/, nhunlth 5 09.09.2020: 


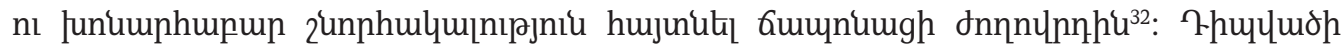

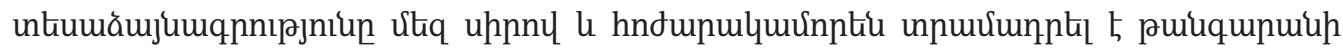

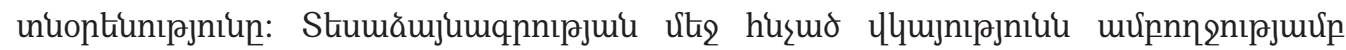
ukplumugruntu tiup unnplis3.

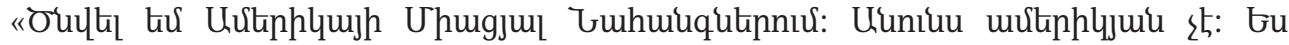

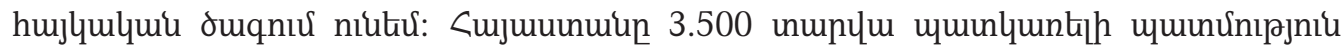

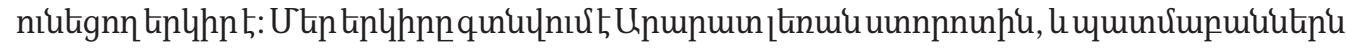

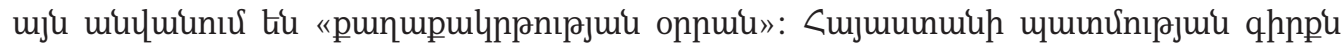

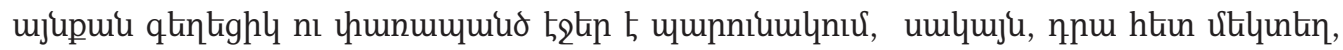

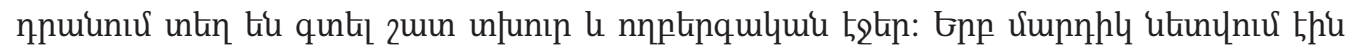

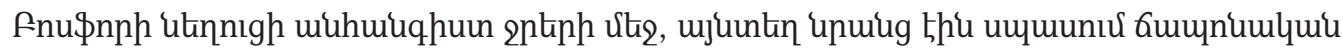

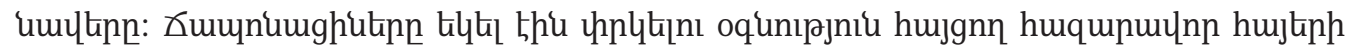

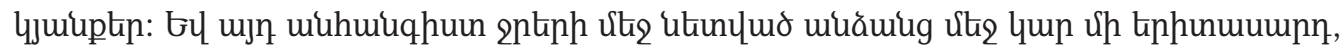

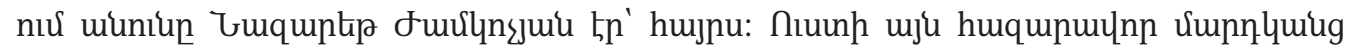

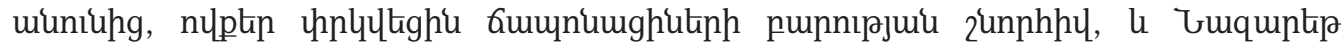

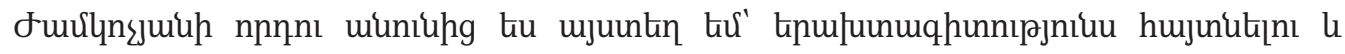

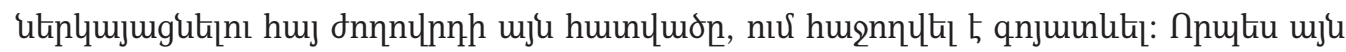

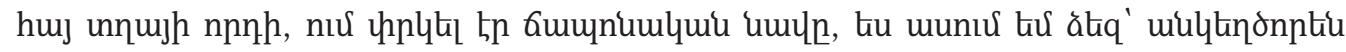
zunphulum tiư ${ }^{34}$ :

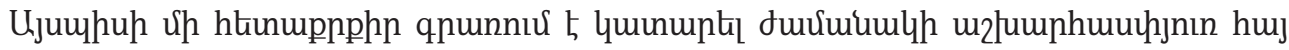

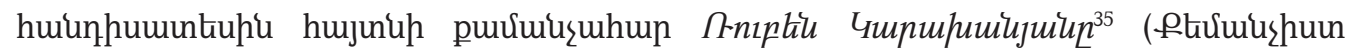

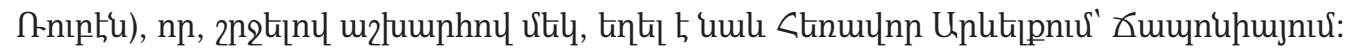

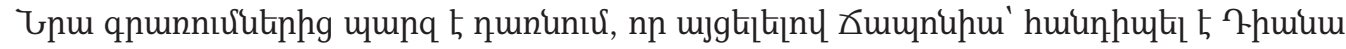
Upqunhu' ${ }^{36}$ :

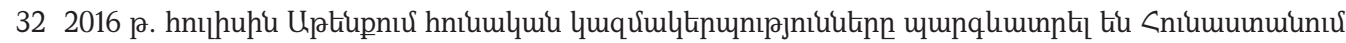

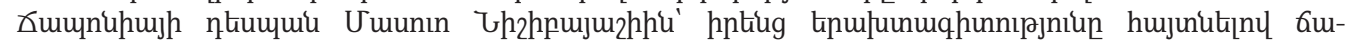

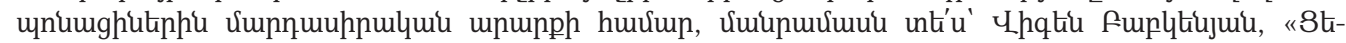

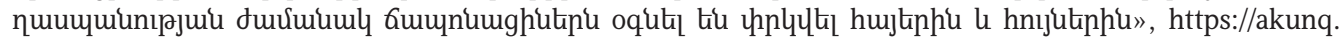
net/am/?cat=22, nhunцt t 506.10.2020:

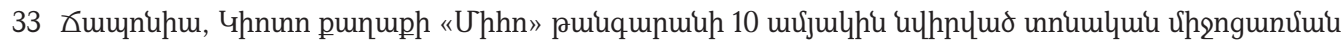
untumămujumqnnıpjnıน:

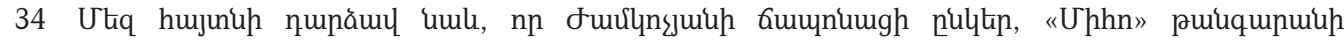

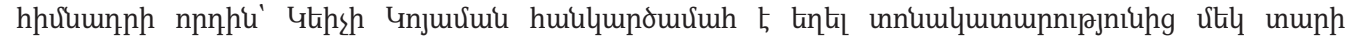

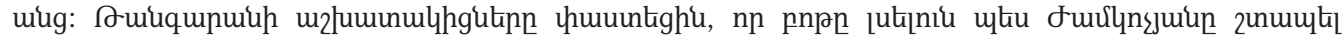

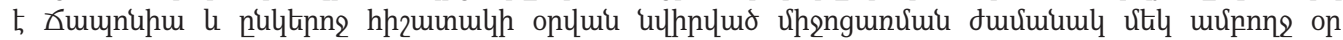

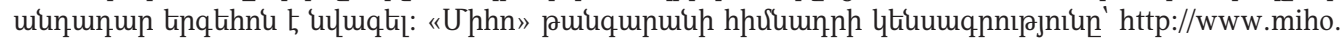
or.jp/en/intro/founder/, nhunltil 5 06.10.2020:

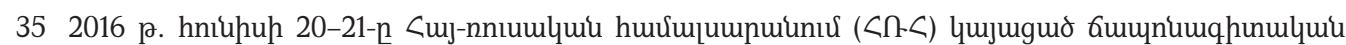

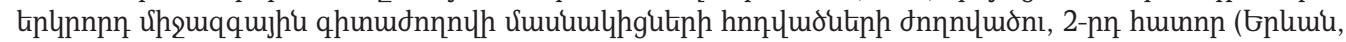
$<\cap<$ hpuununuls nıрjniu, 2017), 113:

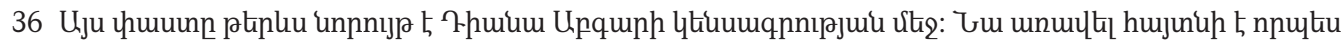

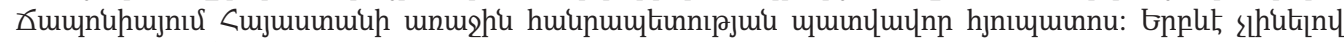

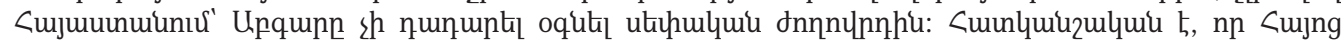

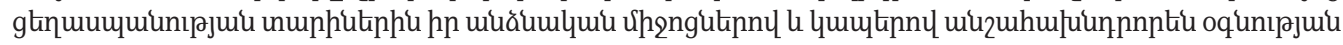




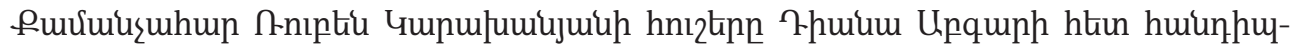

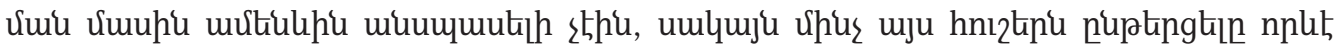

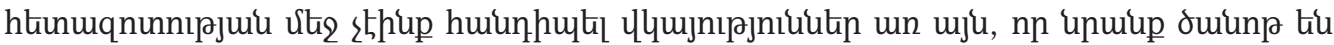

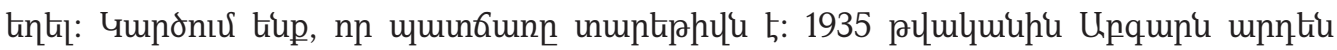

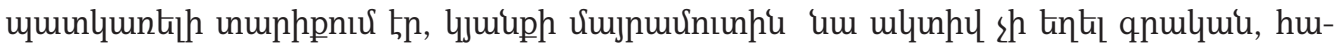

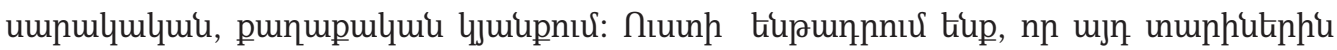

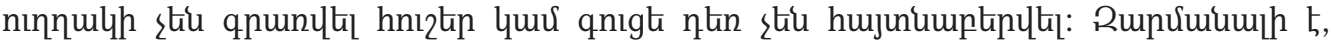

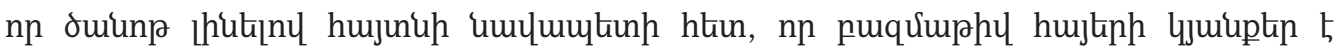

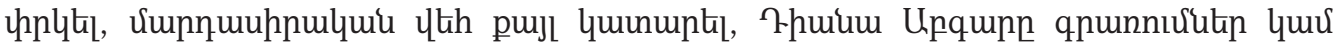

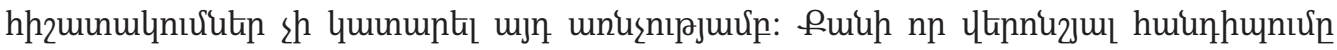

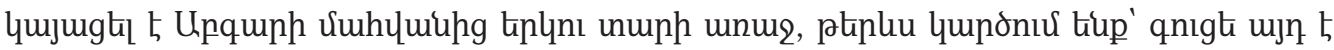

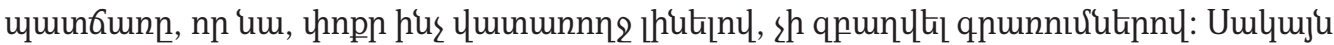

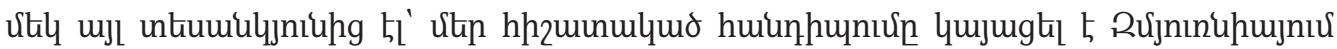

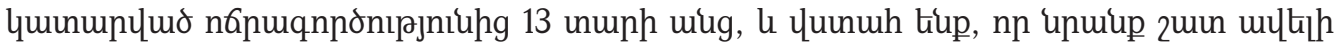

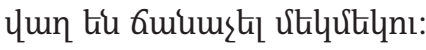

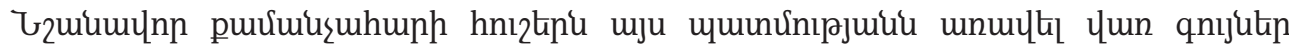

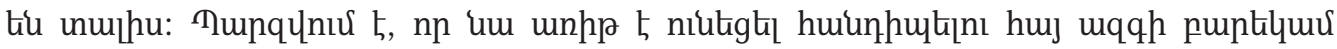

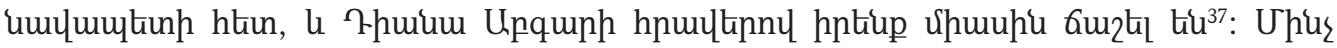

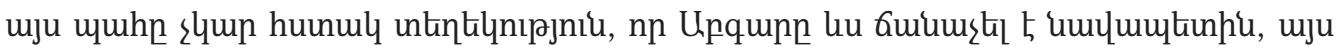

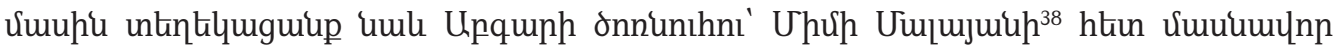

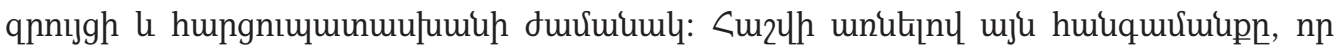

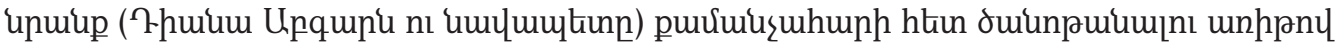

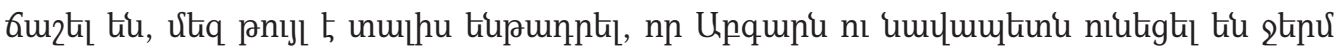

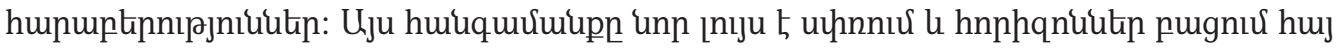

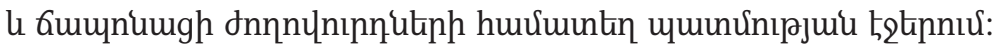

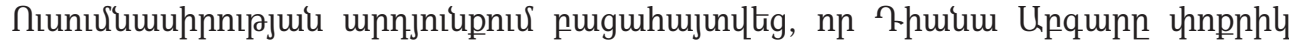

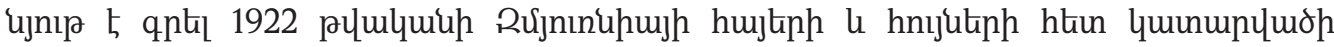

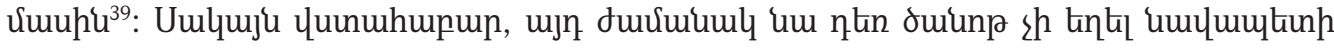

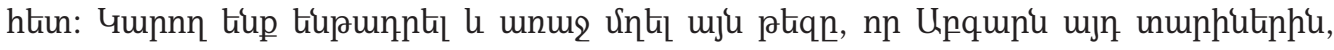

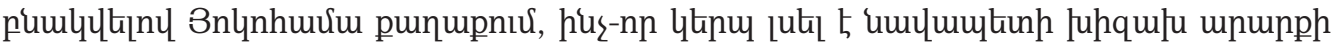

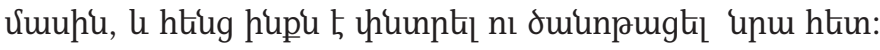

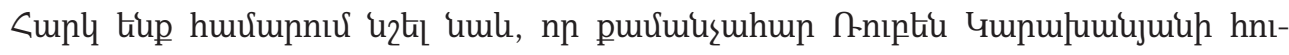

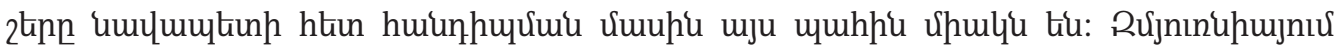

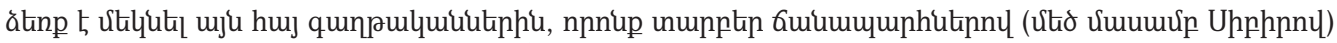

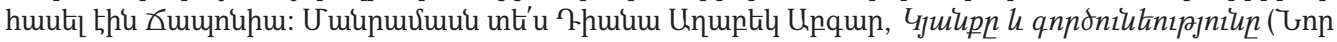
Rnınu, Uumbumuh Cuung ptiu, 2011):

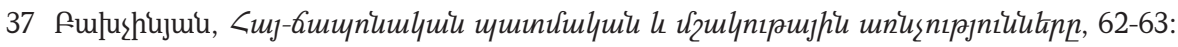

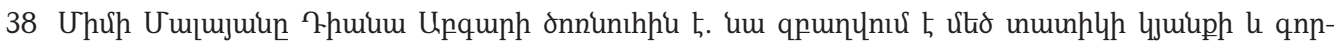

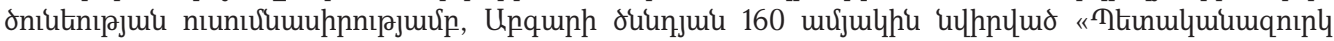

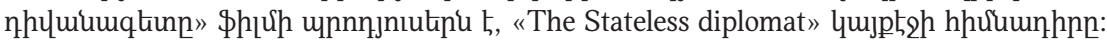

39 Lucille Apcar, From the Book of One Thousand Tales: Stories of Armenia \& Its People 1892-1922, by Diana Agabeg Apcar (USA, Bloomington: AutherHouse, 2004), 123-125. 


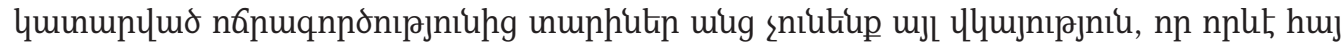

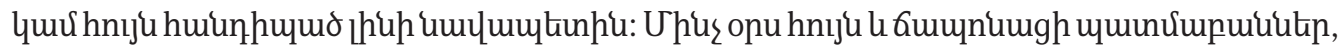

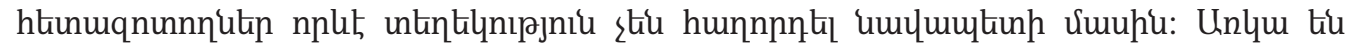

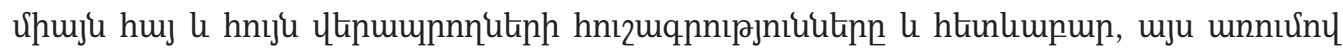
utippnzunumnjul hnı2tinn punănundtip tu:

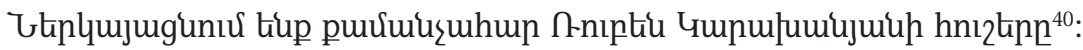

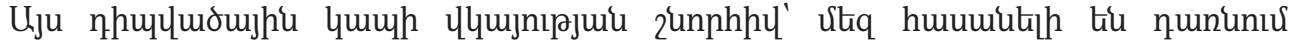

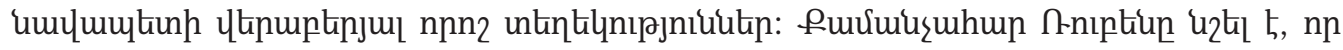

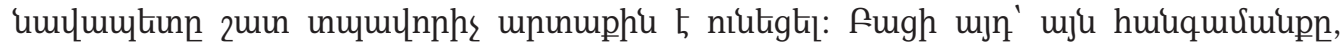

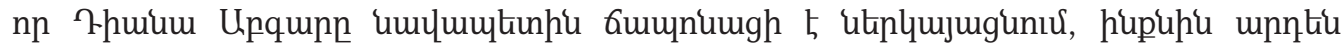

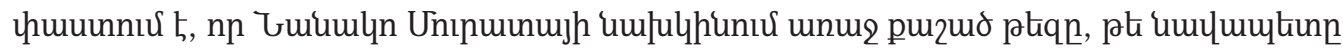

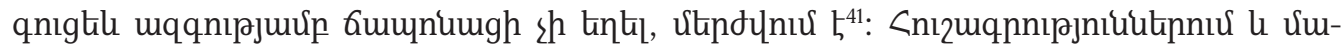

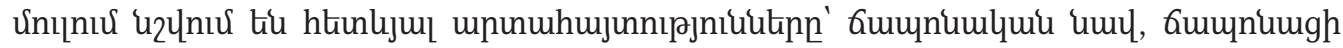

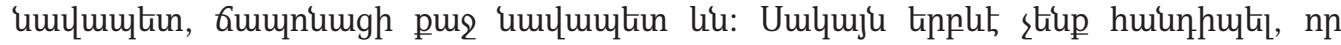

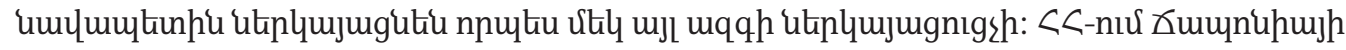

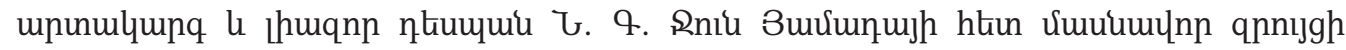

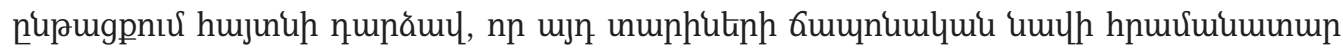
ujl uqqh ukplqujugnıghs sth lupnn thukl:

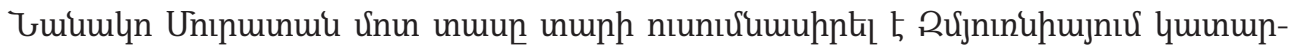

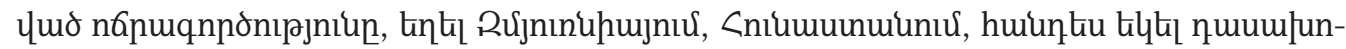

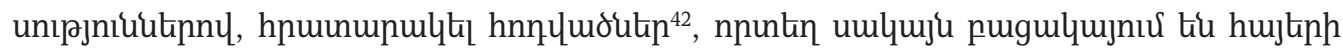

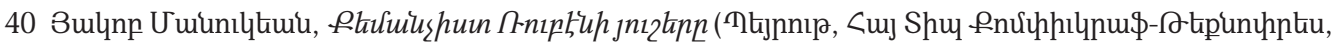
1989), 60-61:

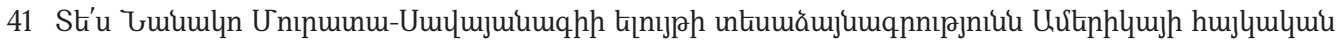
uqqujhu lnưhunth (Armenian National Committe America) Uh@ngunưuu duưuuuul, 2019 https://www.

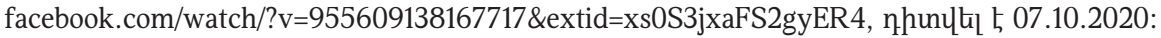

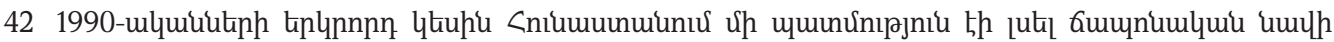

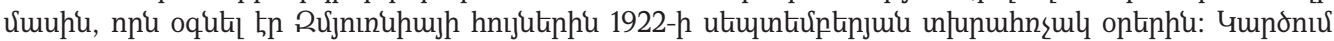

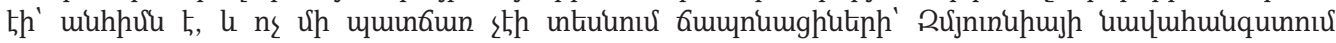

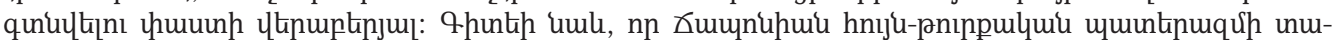

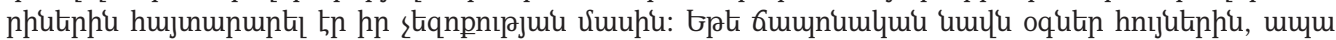

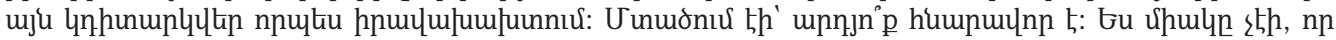

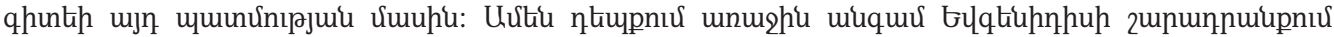

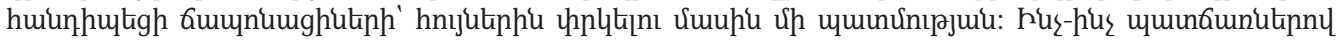

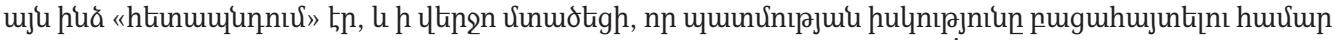

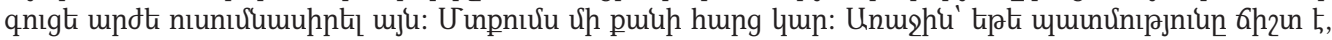

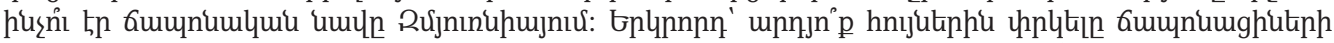

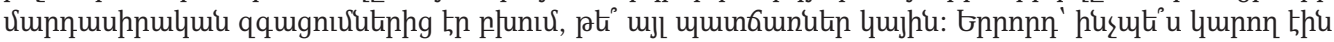

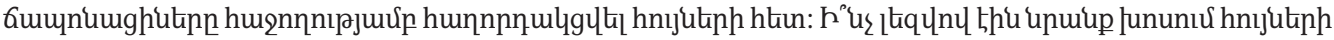

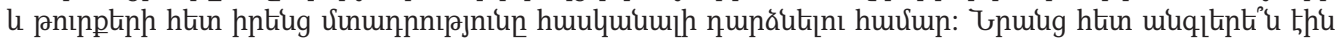

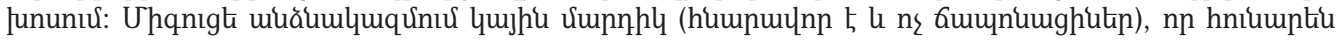

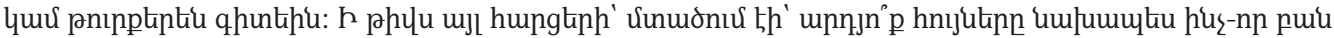

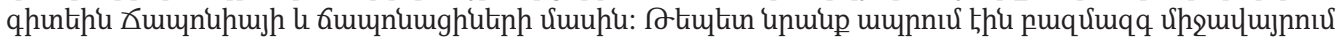

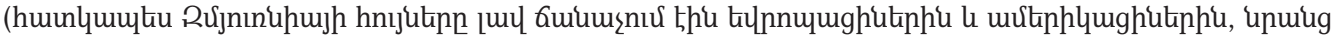

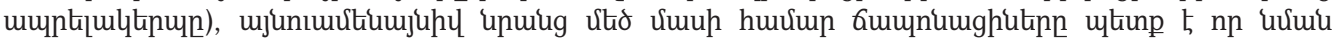

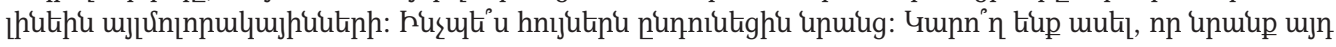


Umuhu untintilnıpjniuutinn:

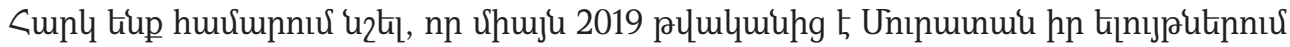

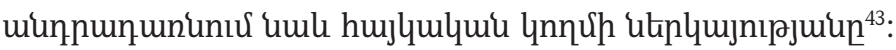

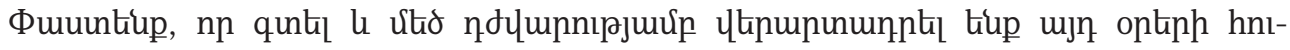

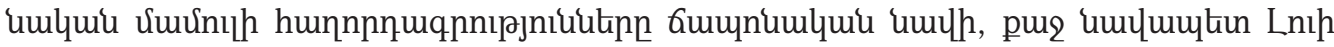

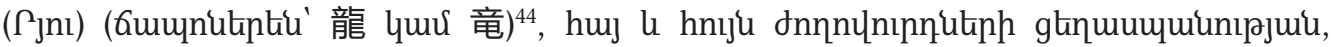

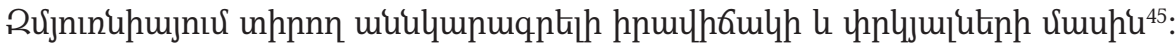

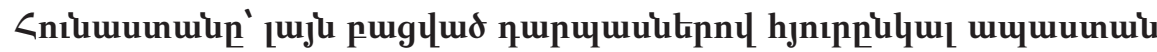

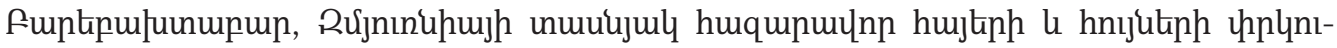

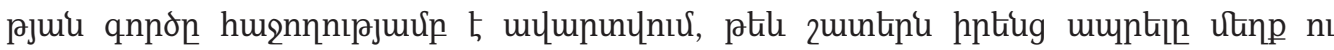

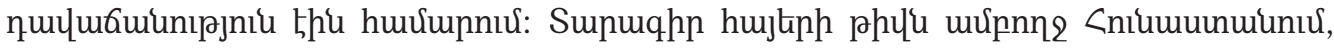

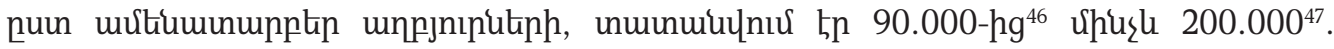

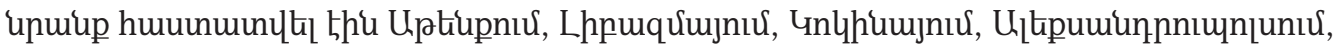

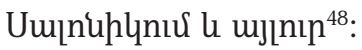

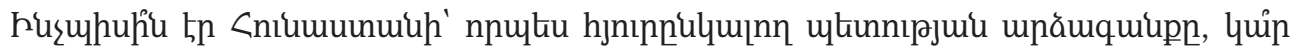

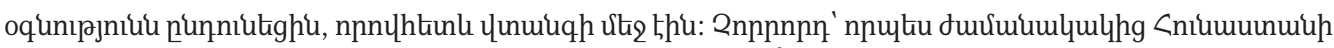

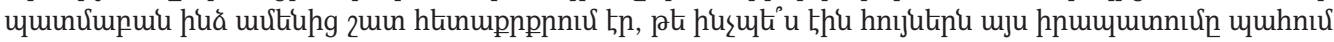

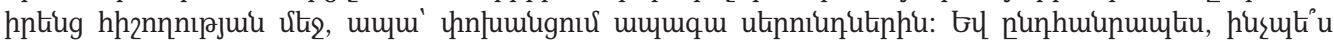

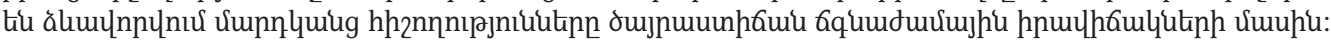

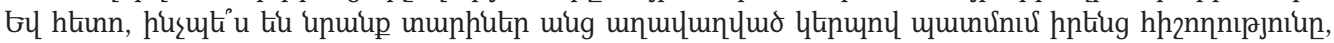
Nanako Murata-Sawayanagi, "The Memory in a Crisis: A Japanese Ship Helping Out Greek Refugees on the Quay of Smyrna in 1922," at https://hermes-ir.lib.hit-u.ac.jp/hermes/ir/re/28545/?lang=1, nhunltal b 07.10.2020:

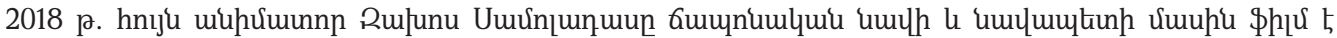

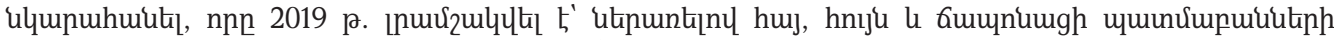
htunuqnunnıjniuutiph upnjniuputinn, unt'u' https://www.1tv.am/hy/video/\%D4\%B2\%D5\%A1\%D6\%81\% D5\%A1\%D5\%B0\%D5\%A1\%D5\%B5\%D5\%BF\%D5\%AB\%D6\%80-\%D5\%80\%D5\%A1\%D5\%B5\%D5\%A1\%D5\%BD\% D5\%BF\%D5\%A1\%D5\%B6\%D5\%A8-\%D5\%93\%D5\%B8\%D6\%84\%D6\%80-\%5\%A1\%D5\%A6\%D5\%A3\%D5\%AB\%D5\%B4\%D5\%A5\%D5\%AE-\%D5\%BA\%D5\%A1\%D5\%BF\%D5\%B4\%D5\%B8\%D6\%82\%D5\%A9\%D5\%B5\%D5\%B8\%D6 \%82\%D5\%B6/167631?fbclid=IwAR212EwtbDAcpsr8mBBEqGISzKK271DaXFGSMsilNXohpYkUgmg0xMgaK1w, nhunltal 5 10.12.2020:

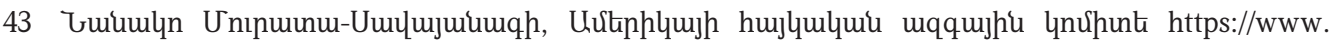
facebook.com/watch/?v=955609138167717\&extid=xs0S3jxaFS2gyER4, nhunцth t 07.10.2020:

44 Sti'u' https://myoji-yurai.net/searchResult.htm?myojiKanji=\%E9\%BE\%8D, https://myoji-yurai.net/ searchResult.htm?myojiKanji=\%E7\%AB\%9C, https://www.youtube.com/watch?fbclid=IwAR3OcYxPLvHg TWvQ9WFCSLtjWXjuH-wk2WN1xpd-6jhccZnn_1ZeEQTYosg\&v=Yc-FGVi1B1M\&app=desktop, nhunцl 10.10.2020:

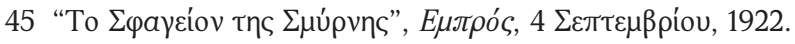

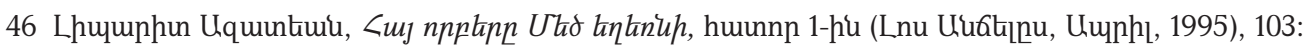

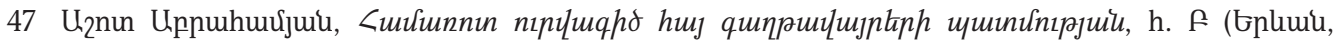
Cujulkinhpuiu, 1967), 105:

48 七nıju untinnư: 


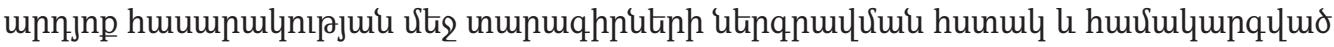

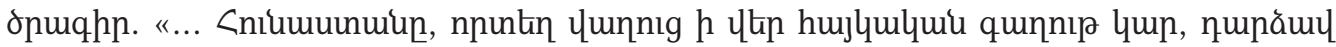

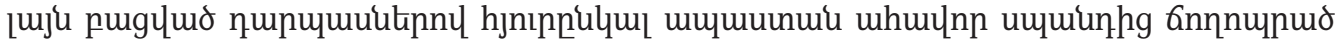

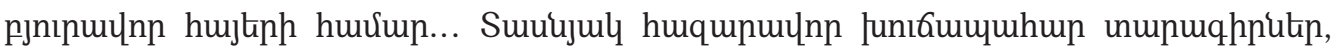

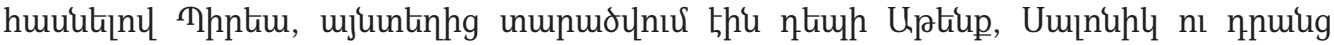

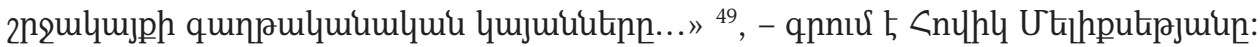

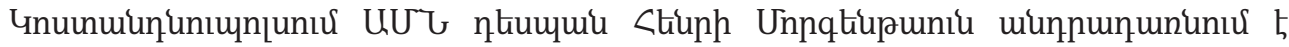

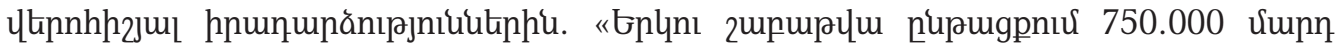

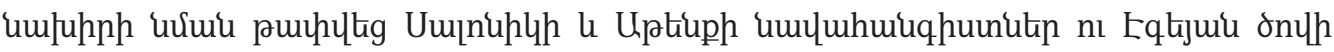

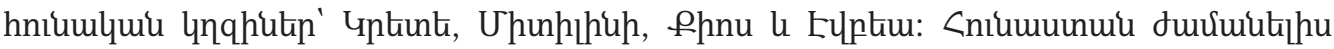

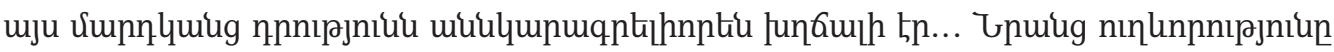

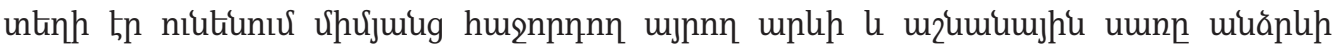

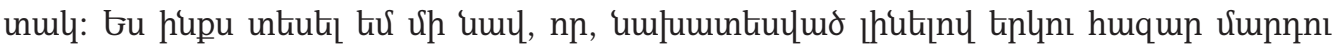

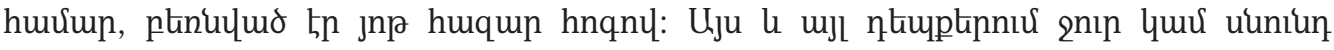

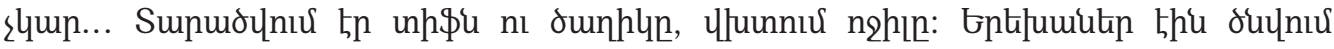

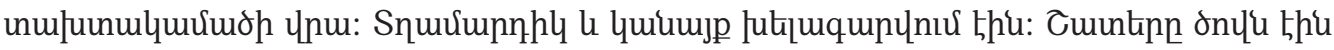

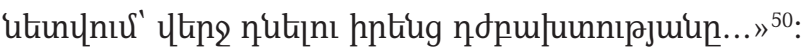

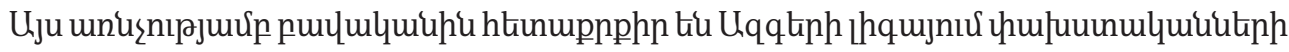

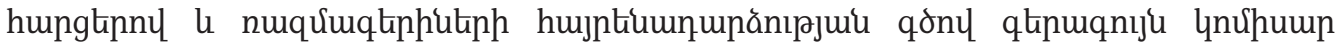

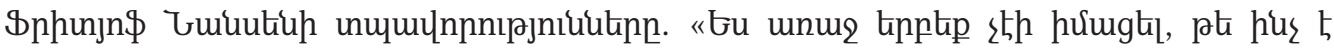

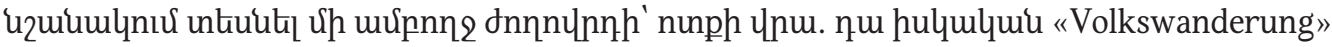

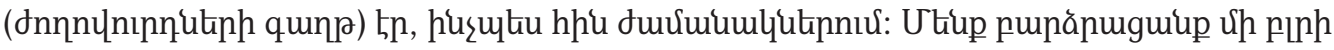

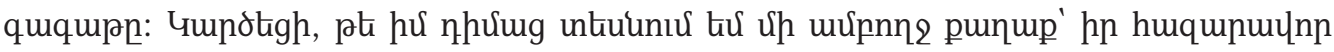

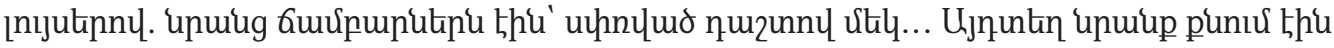

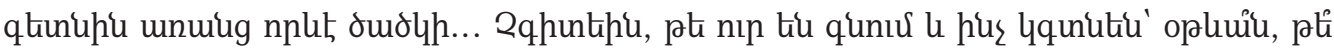
P2Чunnıpjniu» ${ }^{51}$ :

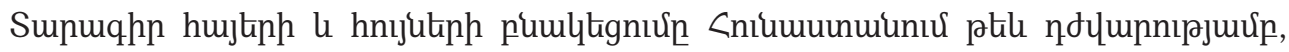

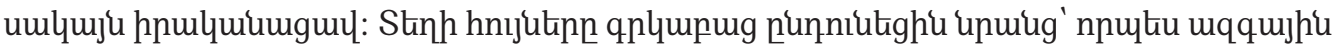

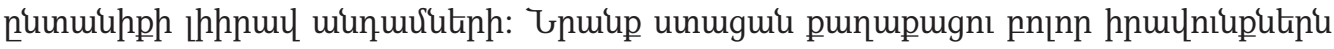

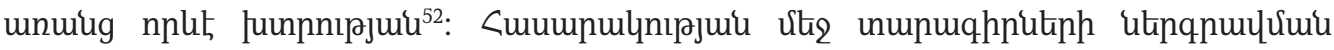

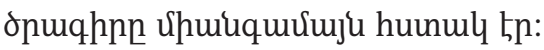

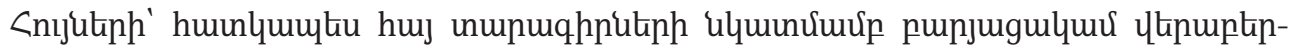

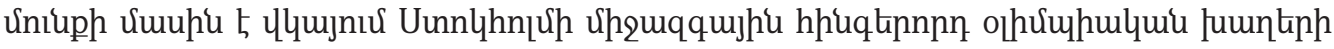

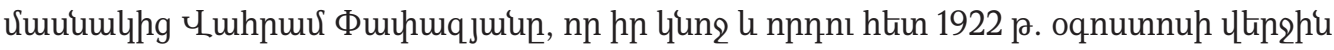

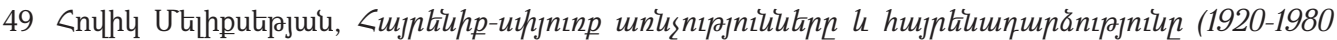

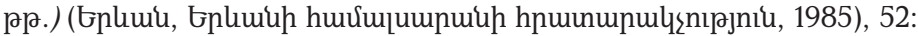

50 Henry Morgenthau, I was Sent to Athens, in collaboration with French Strother (New York, Doubleday: Doran \& Company, 1929), 47- 49.

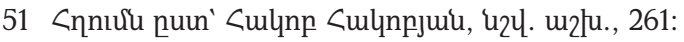

52 Morgenthau, I was Sent to Athens, 104. 


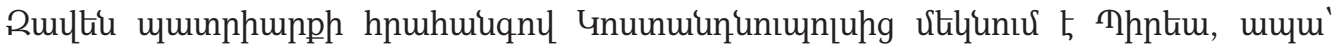

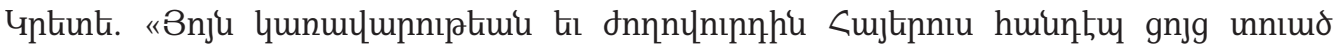

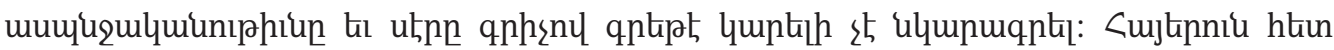

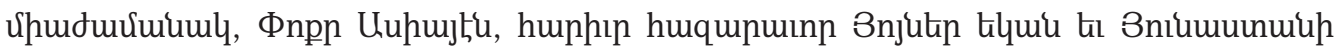

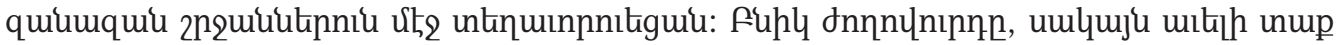

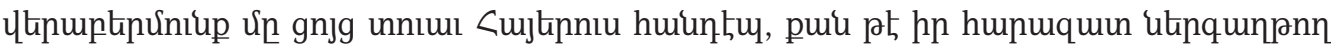

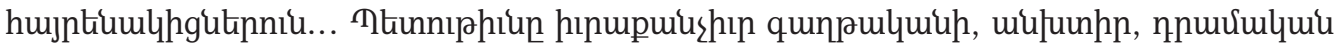

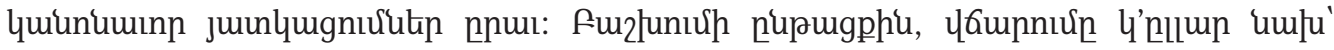

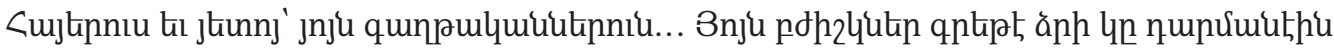

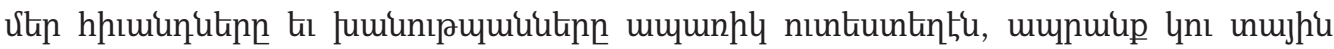

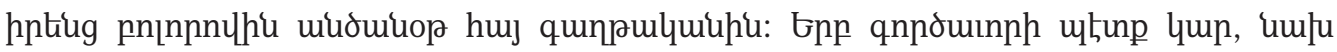

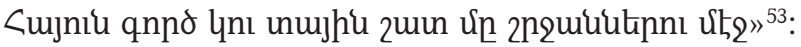

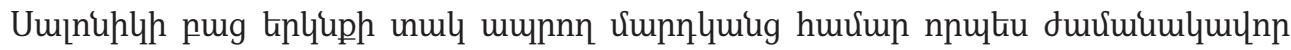

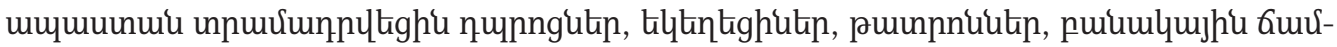

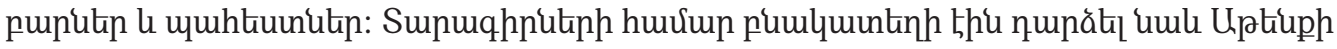

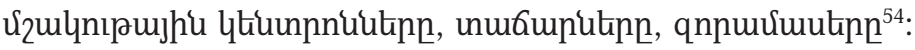

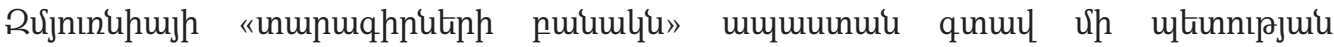

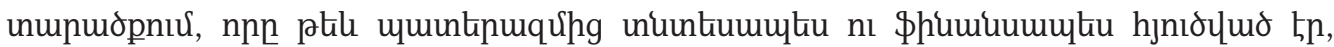

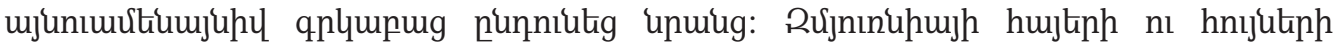

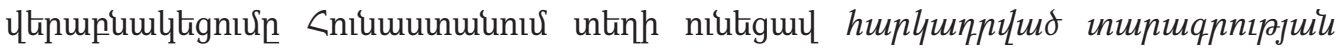

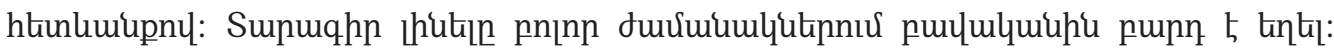

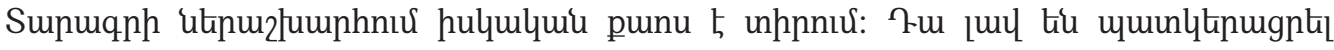

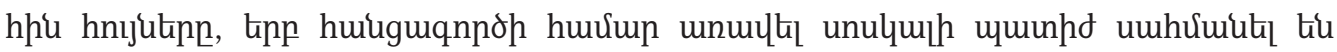

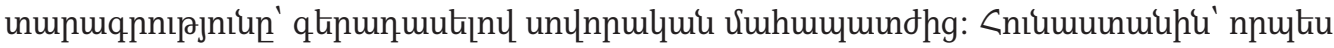

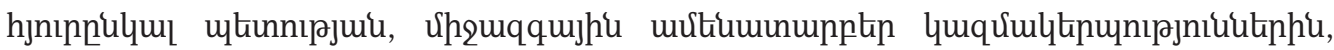

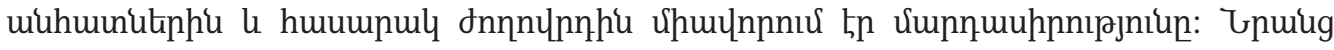

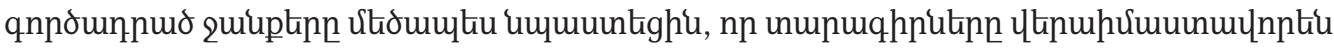

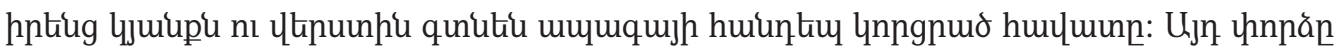

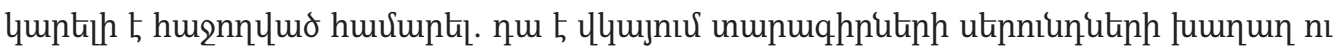
uuntinduqnno qnjulggnıрjniup ${ }^{55}$ :

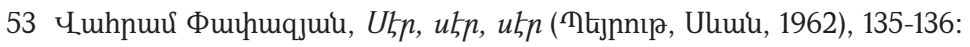

54 James L. Barton, Story of Near East Relief (1915-1930) (New York: the Macmillan Company, 1930), 162.

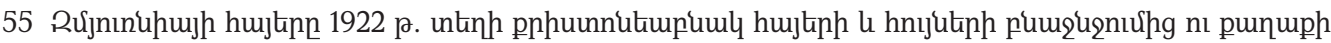

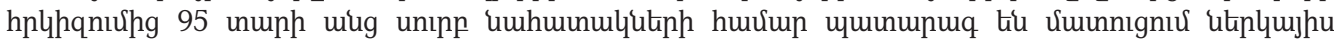
hqưhnnư, unt'u' https://www.tert.am/am/news/2017/08/15/izmir/2457941?fbclid=IwAR3BXISIW0Nc32DH gqAWjot1HFXaz0I_vkh-uUB6BvaPIQ1EIutAN_X_kxk, nhunцlıl 5 10.10.2020: 


\section{<Uप,GLU.Uర56}

\section{Guiuutiunp}

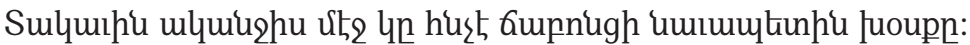

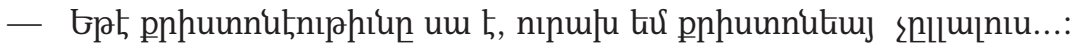

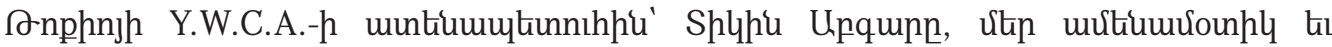

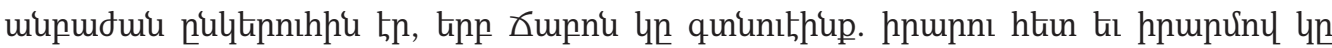

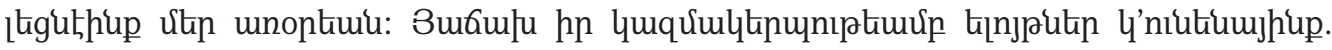

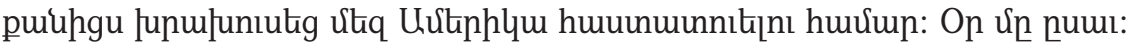

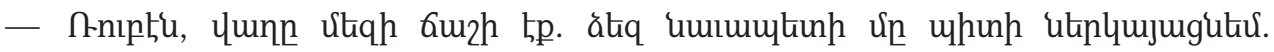
htunuppphp dtpniuh uni Ł, qnh l'nllup:

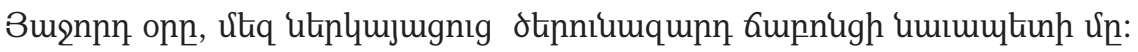

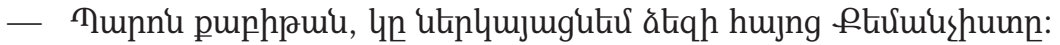

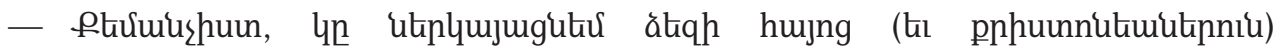

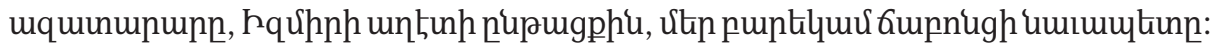

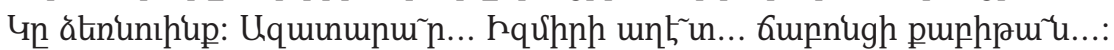

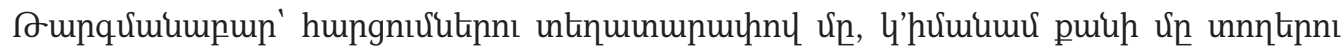

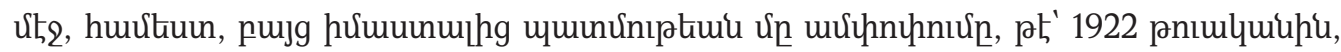

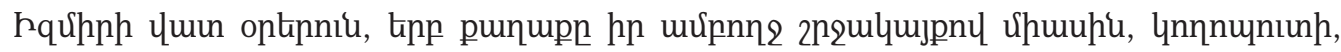

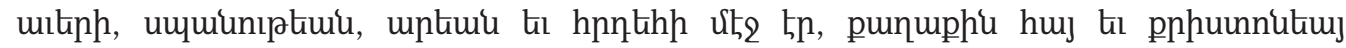

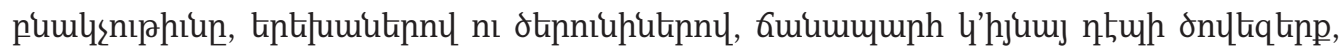

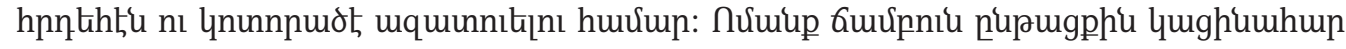

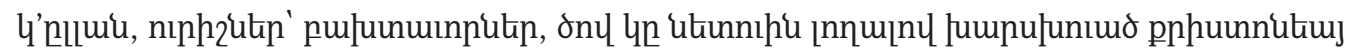

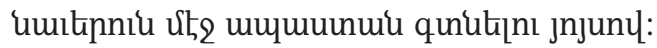

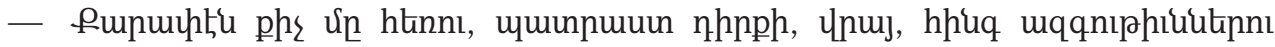

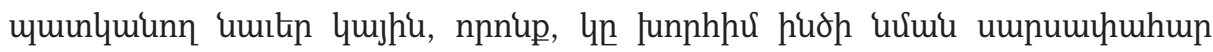

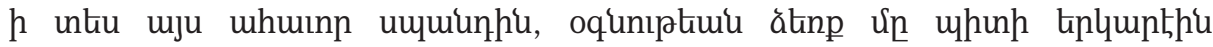

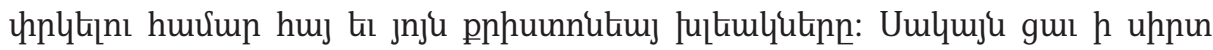

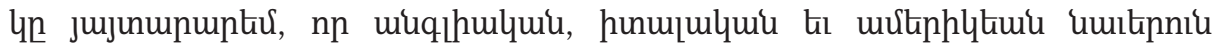

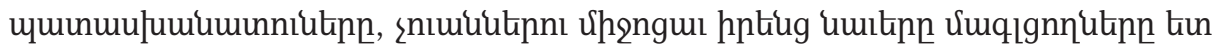

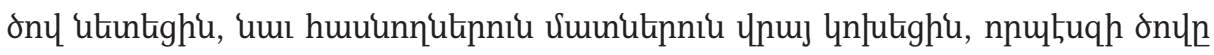

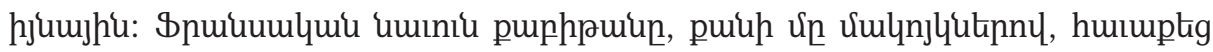

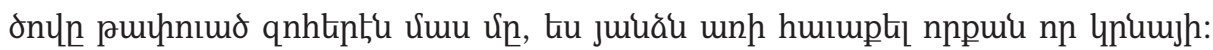

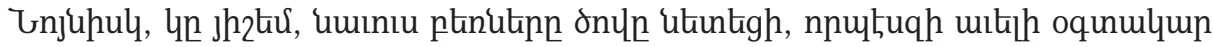

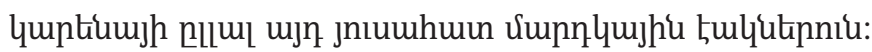

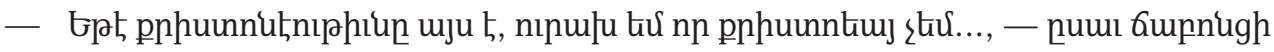
uuiuuutiunn:

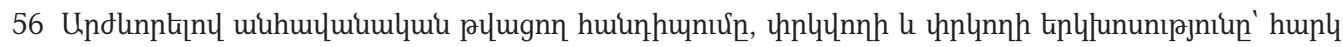

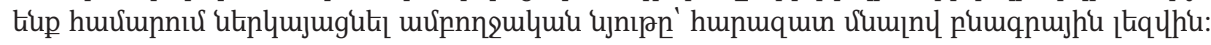




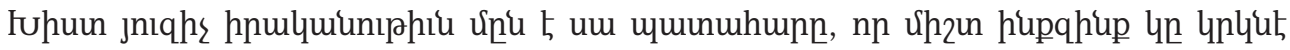

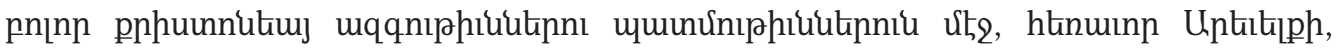

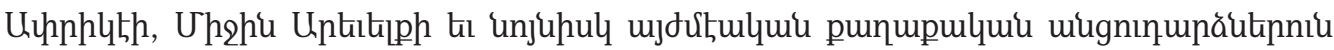

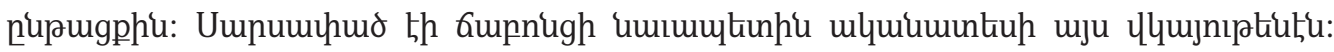

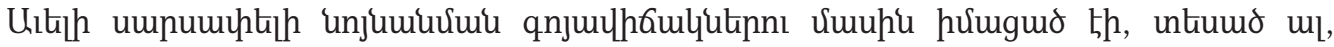

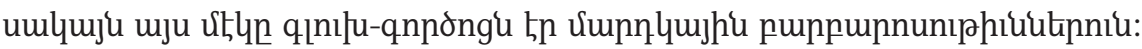

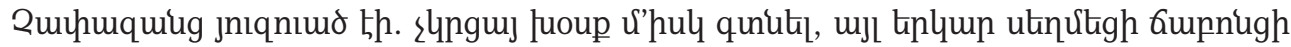

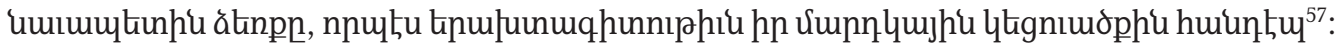

Anna A. Vardanyan

$\mathrm{PhD}$ in Economics

Tehmine R. Martoyan

$\mathrm{PhD}$ in History

TRACKING THE JAPANESE SHIP:

\section{THE RESCUE ROUTE OF SMYRNA'S ARMENIANS AND GREEKS}

\section{SUMMARY}

Keywords: Armenian Genocide, Greek Genocide, exile, Japanese ship, captain, rescue, humanitarianism, Smyrna, Greece.

The purpose of the research is to comprehensively present the rescue process of the Armenians and Greeks exiled from Smyrna, tracking the example of a Japanese ship. To achieve the stated goal, the history of the Smyrna fire and the extermination of the Armenian and Greek populations of the local Christian districts have been studied, the details of the Japanese ship's arrival and the passage of the survivors to the Greek shores has been explored according to the verificated data by eyewitness-survivors.

The relevance of the research topic is conditioned by the "originality" and importance of the rescue operation performed by the Japanese ship. Voicing out about such realities nowadays will contribute educating a righteous and unbiased society.

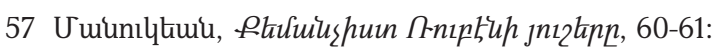


Анна А. Варданян

кандидат экономических наук

Тегмине Р. Мартоян

кандидат исторических наук

\section{ЗА ТРАЕКТОРИЕЙ ЯПОНСКОГО КОРАБЛЯ: ПУТЬ СПАСЕНИЯ АРМЯН И ГРЕКОВ СМИРНЫ}

\section{РЕЗЮМЕ}

Ключевые слова: Геноцид армян, Геноцид греков, депортация, японский корабль, капитан, спасение, человечество, Смирна, Греция.

Целью исследования является полное представление процесса спасения депортированных армянских и греческих беженцев в Смирне на примере японского корабля. На фоне истории поджога Смирны, истребления армяно-греческого населения в местных христианских кварталах, раскрыты подробности прибытия японского корабля, спасения сотен выживших и их доставки к греческому берегу.

Актуальность темы исследования обусловлена «оригинальностью» и важностью спасательной операции японского корабля. Освещение таких реалий в наши дни поможет воспитать благочестивое и альтруистическое общество.

\section{REFERENCES}

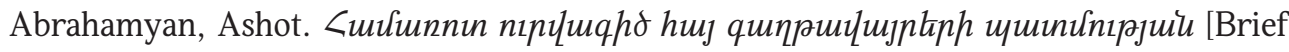
Outline of the History of Armenian Colonies]. Yerevan: Haypethrat, 1967;

Azadian, Libarid. <uj nnpknn Utid kqkniuh [The Orphans of the Armenian Genocide]. Los Angeles: April, 1995;

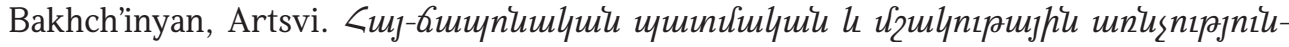
utann [Armenian-Japanese Historical and Cultural Connections]. Yerevan: Author's edition, 2018;

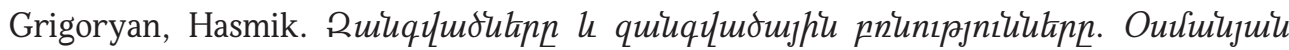

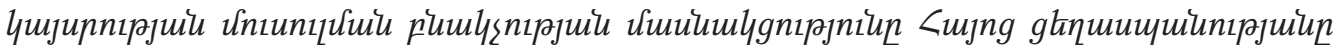
[The Masses and Mass Violence: Participation of Muslim Population of the Ottoman Empire in the Armenian Genocide].Yerevan: Gitut'yun, 2017;

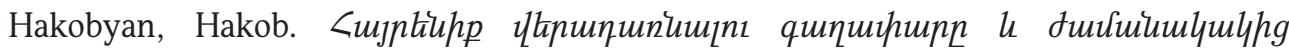

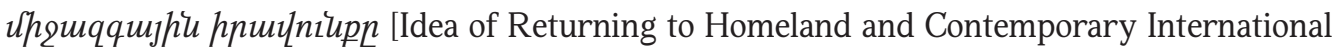
Law]. Yerevan: Asoghik, 2000;

Manoukian, Hakob. Pkukiushuin Nnıp5uh jnızknn [Memories of Qemanch'ist Rouben]. Beirut: High Type Compugraph-Technopress S.A.L., 1989; 
Lйиш U. Ч ширпшијші

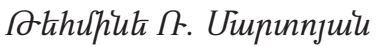

Martoyan, Tehmine. «Rujnınupujh huj lu hnıju pumlınıpjuiu pumququiuu

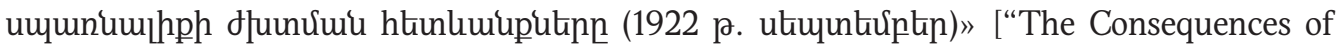
the Denial of the Threat of Extermination in Case of the Greek and the Armenian Population of Smyrna (September 1922)"], Hayagitutyan harcer no. 1 (2014): 61-75;

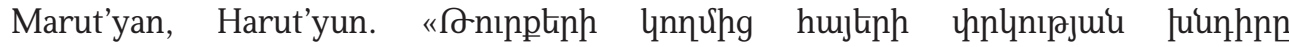

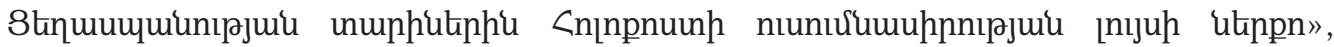

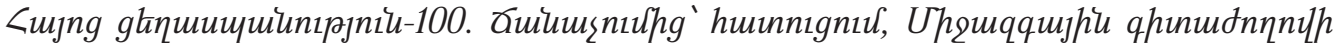
qkilnigniluknh qnnijplutin ["The Question of Rescuing Armenians During the Genocide in the Light of the Holocaust Researches," in Armenian Genocide-100. Collection of Papers of the International Conference]. Yerevan: Gitut'yun, 2016, 218-221;

Marut'yan, Harut'yun. "Lujng gtnuuщuinnрjui lu Cntimutiph hnjnpnuinh

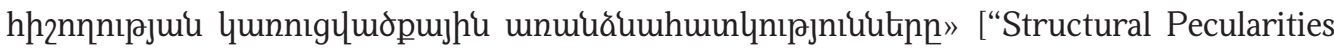
of Armenian Genocide and Jewish Holocaust Memory"]. Patmabanasirakan handes 2 no. 187(2011): 24-46;

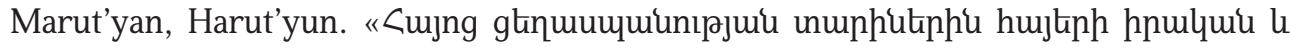

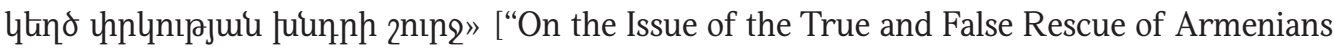
during the Armenian Genocide"]. Lraber hasarakakan gitutyunneri no. 2 (2018): 30-60;

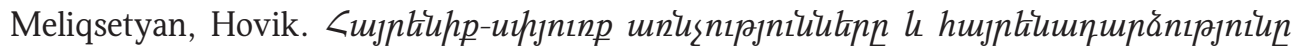
(1920-1980 рр.) [Homeland-Diaspora Relations and Repatriation]. Yerevan: Yerevan University, 1985;

Papazyan, Vahram. $U 5 n, u 5 n$, uhn [Love, Love, Love]. Beirut: Sevan, 1962;

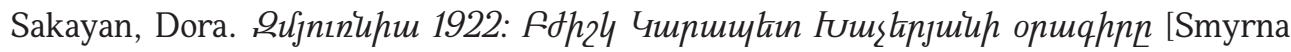
1922, Armenian Doctor Garabed Hatcherian's Diary]. Yerevan: Nahapet, 2005;

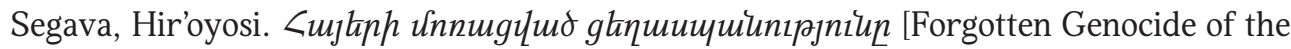
Armenians]. Yerevan: Nshanak, 2005;

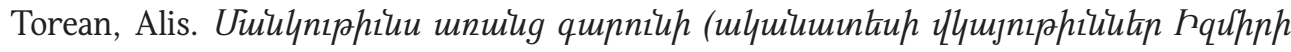
un 5 n 5 L) [My Childhood without Spring (Eye-witness Testimonies from Izmir Catastrophe)]. Beiruit: Atlas, 1975;

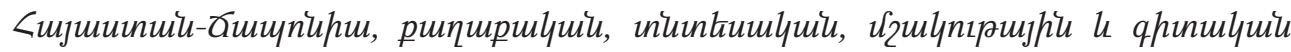
hupupknnipjniliukn [Armenia-Japan, Political, Economic, Cultural and Scientific Relations]. Yerevan: Zangak, 2005. 


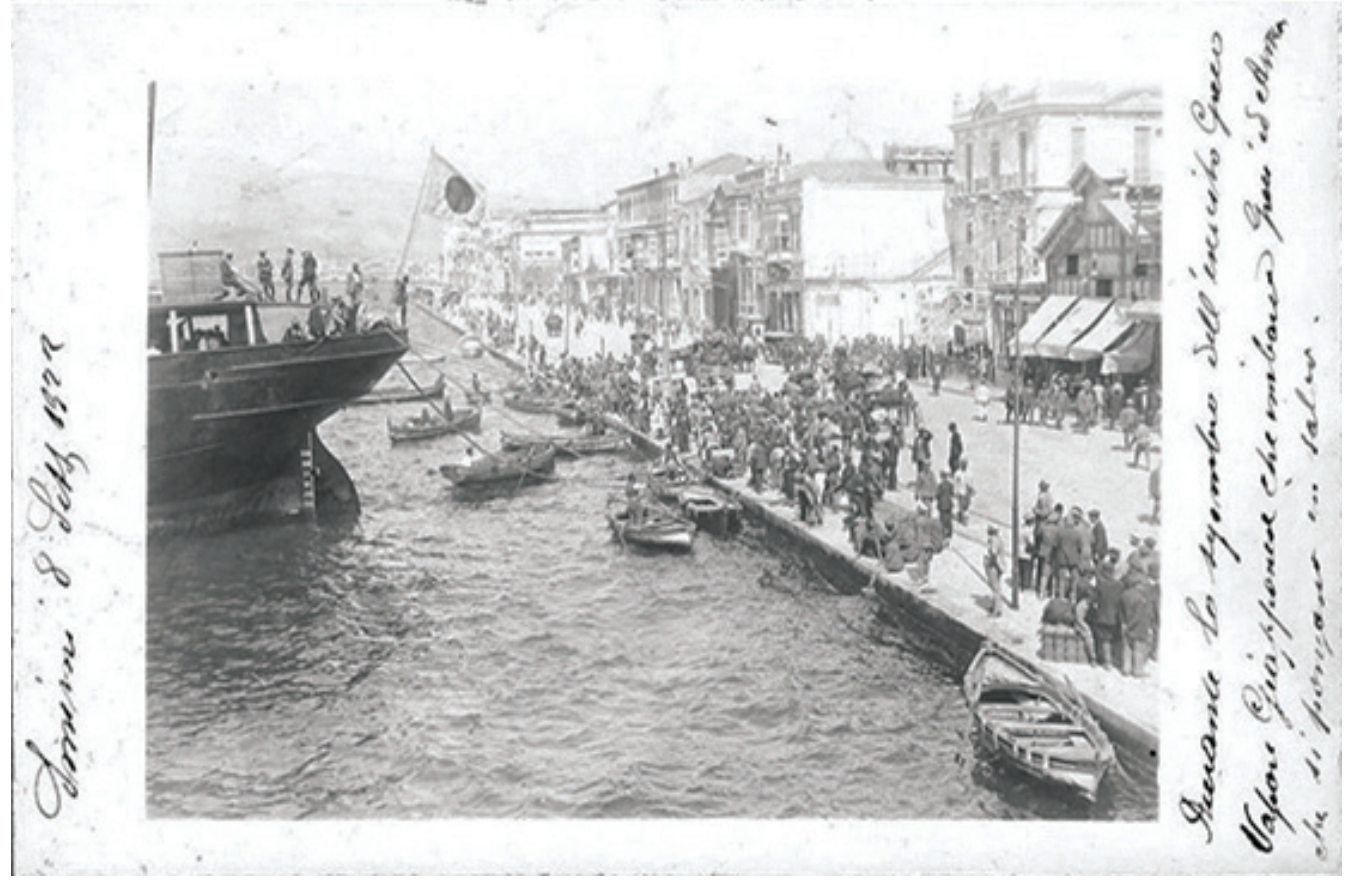

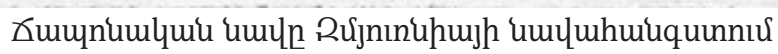

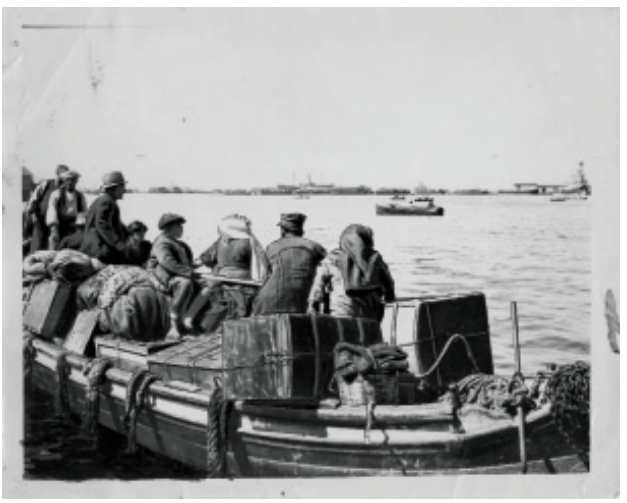

Rujnınuhujh unupuqhputinn

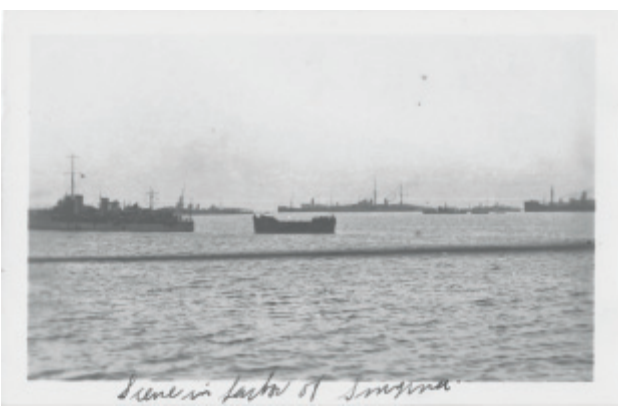

Rर्unınupujh onцuiunn2n

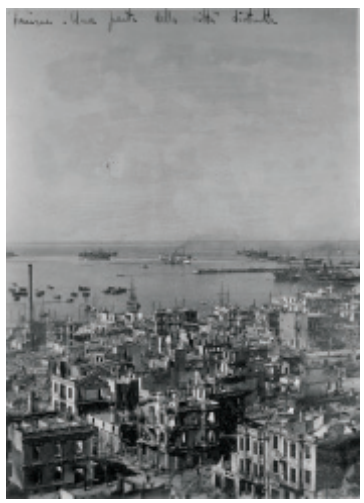

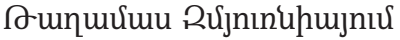

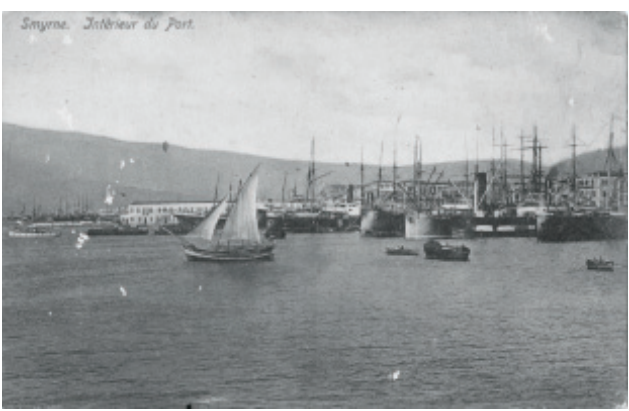

Rujnınuhujh umuluhwiuqhumn 